\title{
Moduli-induced baryogenesis
}

\author{
Koji Ishiwata, ${ }^{a}$ Kwang Sik Jeong ${ }^{a}$ and Fuminobu Takahashi ${ }^{b, c}$ \\ ${ }^{a}$ Deutsches Elektronen Synchrotron DESY, \\ Notkestrasse 85, 22607 Hamburg, Germany \\ ${ }^{b}$ Department of Physics, Tohoku University, \\ Sendai 980-8578, Japan \\ ${ }^{c}$ Kavli IPMU, TODIAS, University of Tokyo, \\ Kashiwa 27r-8583, Japan \\ E-mail: koji.ishiwata@desy.de, kwangsik.jeong@desy.de, \\ fumi@tuhep.phys.tohoku.ac.jp
}

ABSTRACT: We study a scenario for baryogenesis in modular cosmology and discuss its implications for the moduli stabilization mechanism and the supersymmetry (SUSY) breaking scale. If moduli fields dominate the Universe and decay into the standard model particles through diatonic couplings, the right amount of baryon asymmetry can be generated through CP violating decay of gluino into quark and squark followed by baryon-number violating squark decay. We find that, in the KKLT-type moduli stabilization, at least two non-perturbative terms are required to obtain a sizable CP phase, and that the successful baryogenesis is possible for the soft SUSY breaking mass heavier than $\mathcal{O}(1) \mathrm{TeV}$. A part of the parameter space for successful baryogenesis can be probed at the collider experiments, dinucleon decay search experiment, and the measurements of electric dipole moments of neutron and electron. It is also shown that similar baryogenesis works in the case of the gravitino- or the saxion-dominated Universe.

KeYwords: Supersymmetry Breaking, Cosmology of Theories beyond the SM, Supergravity Models

ARXIV EPRINT: 1312.0954 


\section{Contents}

1 Introduction 1

2 Moduli stabilization $\quad 3$

2.1 KKLT mechanism 4

2.2 CP violation in the moduli sector 6

3 Baryogenesis $\quad 8$

3.1 Baryon asymmetry 8

$\begin{array}{ll}3.2 \text { Numerical result } & 13\end{array}$

4 Other scenarios $\quad 16$

$\begin{array}{lll}4.1 \text { Gravitino-induced baryogenesis } & 16\end{array}$

$\begin{array}{lll}4.2 & \text { Saxion-induced baryogenesis } & 18\end{array}$

5 Conclusions and Discussion $\quad 21$

\section{Introduction}

The origin of baryon asymmetry is one of the most profound puzzles in modern cosmology and particle physics. It is well-known that baryon accounts for about $5 \%$ of the total energy density of the Universe [1]:

$$
n_{B} / s=(8.6 \pm 0.1) \times 10^{-11}(68 \% \text { C.L. }),
$$

where $n_{B}$ is the number density of baryon, and $s$ is the entropy density. The standard model (SM) of particle physics and standard cosmology, however, do not provide any viable mechanism to generate the observed baryon number. To be specific, three conditions known as the Sakharov conditions [2] have to be satisfied for successful baryogenesis: (i) baryon number violation, (ii) $\mathrm{C}$ and $\mathrm{CP}$ violation, and (iii) departure from thermal equilibrium. These conditions require physics beyond the SM and set tight constraints on possible scenarios for baryogenesis.

The mechanism for baryogenesis is closely related to the thermal history of the Universe. The recent observations of cosmic microwave background as well as large scale structure of the Universe firmly support the inflationary Universe in which our Universe experienced an accelerating cosmic expansion at an early stage of the evolution [3-7]. Thus baryon asymmetry must be generated after inflation since otherwise the exponential expansion during inflation would completely dilute any pre-existing baryon asymmetry.

However, the history of the Universe after inflation is poorly known. The inflaton may directly decay into the SM particles to reheat the Universe. The right amount of baryon 
asymmetry can be generated through thermal leptogenesis if the reheating temperature is sufficiently high [8]. On the other hand, the evolution of the Universe could be more involved. Indeed, there are many moduli fields in supergravity and superstring theories, which may have a significant effect on the thermal history. They are known to easily dominate the energy density of the Universe because the moduli fields are copiously produced by the coherent oscillation and that their interactions are extremely weak, suppressed by the Planck scale or Grand Unified Theory (GUT) scale. Then a huge amount of entropy is released by the moduli decay, diluting pre-existing baryon asymmetry. As a consequence we have two possibilities for baryogenesis. One is to create a sufficiently large amount of baryon asymmetry before the moduli decay by, e.g., the Affleck-Dine mechanism [9, 10], which has been extensively studied in a context of modular cosmology [11-16]. The other is to generate baryon asymmetry after the moduli decay. This is the main focus of the present paper.

We assume that moduli fields dominate the energy density of the Universe in the framework of supergravity. After dominating the Universe, moduli decay to lighter particles in the minimal extension of supersymmetric standard model (MSSM). The moduli decay temperature can be higher than $\mathcal{O}(1) \mathrm{MeV}$ for the moduli mass heavier than about $10 \mathrm{TeV}$ if their interactions are suppressed by the Planck mass, thus avoiding bounds from the big bang nucleosynthesis (BBN) [17-20]. In this paper we study a baryogenesis scenario in which the baryon asymmetry is generated through $\mathrm{CP}$ violating decay of gluino into quark and squark, followed by baryon-number violating squark decay. We will introduce renormalizable R-parity violating interactions to get the required baryon number violation. Late-time moduli decay can easily realize the out-of-equilibrium decays of gluino and squark. On the other hand, the size of the CP phase depends on how supersymmetry (SUSY) breaking is transmitted to the visible sector. As we will see shortly, the required $\mathrm{CP}$ violation for the baryogenesis has important implications for moduli stabilization. Successful baryogenesis is possible in moduli stabilization such that moduli-mediated SUSY breaking is sizable, and the axionic shift symmetry associated with the modulus is broken at least by two terms in the modulus superpotential. We will focus on mixed modulusanomaly mediation in the Kachru-Kallosh-Linde-Trivedi (KKLT)-type moduli stabilization [21], as it provides a natural framework for the moduli-induced baryogenesis. We will also show that similar baryogenesis works in other cases where the gravitino, the saxion, or the Polonyi field dominates the Universe, and decays mainly into the SM gauge sector.

Let us mention differences of the present paper from the works in the past. Baryogenesis induced by a modulus-like particle has been studied since long time ago. The gravitino-induced baryogenesis was proposed by Cline and Raby [22], followed by Mollerach and Roulet who studied a similar scenario where the saxion plays the role of the gravitino [23]. In ref. [24], baryogenesis via a hidden sector inflaton was discussed. Recently baryogenesis via such late-decaying particles was studied in a generic way by with higher dimension operators [25]. In those studies the origin of the CP phase was simply assumed to be independent of the decaying particle. In the present paper we study a baryogenesis within a concrete moduli stabilization mechanism, where the modulus field mediates SUSY breaking with a non-vanishing CP phase, and its decay provides out-of-equilibrium 
decays of gluino and squark. Therefore the moduli-induced baryogenesis studied in this paper offers a realistic and self-contained scenario. Later in this paper we will revisit the gravitino-induced baryogenesis. Here we will provide analytic formulae which can be applied to a more generic mass spectrum of superparticles. Note that it was shown that the inflaton [26-31] as well as moduli [32-35] generically decays into a pair of gravitinos. Therefore the gravitino-dominated Universe can be realized in a broader scenario than originally assumed. Its cosmological and phenomenological implications have been studied recently in ref. [36]. We will also revisit the saxion-induced baryogenesis. In ref. [23], it was indicated that the saxion decay to gauginos is helicity-suppressed. As clearly shown in ref. [37], however, it is not helicity-suppressed and its rate is generically comparable to that into gauge bosons. Taking into account this fact, we will show that the saxion-induced baryogenesis is more efficient than originally considered before.

The rest of this paper is organized as follows. In section 2 we study mixed modulusanomaly mediation in the KKLT-type moduli stabilization, focusing on how to obtain the $\mathrm{CP}$ violation required for successful baryogenesis. We will then examine the baryogenesis by modulus decay in section 3, and study the implications for the SUSY breaking scale and discuss its experimental consequences. In section 4 we discuss similar baryogenesis by the decay of the gravitino and the saxion. Section 5 is devoted to conclusions and discussion. In this paper we take the reduced Planck mass $M_{P} \simeq 2.4 \times 10^{18} \mathrm{GeV}$ to be unity unless otherwise noted.

\section{Moduli stabilization}

We consider the possibility that late-decaying moduli account for the observed baryon asymmetry of the Universe. To produce baryon asymmetry, we need couplings that violate both baryon number and CP. Baryon number violation arises from R-parity violating interactions while satisfying various experimental constraints, such as neutron-antineutron oscillation, dinucleon decay, and proton decay. The required CP violation, on the other hand, can be induced after SUSY breaking from the R-parity violating interactions. However, generating a nonzero CP phase is nontrivial. It depends on the SUSY breaking mechanism and the moduli stabilization.

As is well-known, anomaly mediation [38-40] (and also gauge mediation) generates flavor and CP conserving soft SUSY breaking terms because it takes place mainly through the SM gauge interactions. The soft terms induced by moduli $F$-terms are also considered to preserve both flavor and CP. Here the flavor conservation is a result of flavor-universal rational numbers called the modular weight, which determines the coupling between moduli and the matter fields in visible sector [41,42], meanwhile CP conserving soft terms are the consequence of axionic shift symmetries associated with the moduli [43]. However, this is true only for a simple KKLT-type moduli stabilization. It turns out that a sizable CP violation can be obtained from the moduli sector if

- Soft SUSY breaking terms receive sizable contributions from the moduli $F$-terms, and 
- Non-perturbative corrections, which generate a modulus potential and break the axionic shift symmetry, involve at least two terms.

We will see that the KKLT scenario is a natural framework for the moduli-induced baryogenesis that works at low temperatures. It will be also shown in the next section that such a sizable $\mathrm{CP}$ violation for baryogenesis could have interesting experimental consequences.

\section{$2.1 \quad$ KKLT mechanism}

In the KKLT compactification [21], all the moduli are stabilized at a phenomenologically viable vacuum. There are three types of moduli; dilaton, complex structure moduli, and Kähler moduli. The dilation and complex structure moduli are fixed by fluxes while obtaining masses hierarchically larger than the gravitino mass. Incorporating non-perturbative corrections to the superpotential, one can also stabilize Kähler moduli at a supersymmetric anti-de Sitter (AdS) minimum. Since our vacuum is a SUSY breaking de Sitter with a tiny cosmological constant, we introduce an anti-brane stabilized at the tip of a highly warped throat. Then its tension provides a small positive energy to cancel the negative cosmological constant. At the same time it explicitly breaks the $N=1$ SUSY preserved by the background geometry and fluxes. To be precise, a small vacuum shift is induced and makes the moduli develop nonzero $F$-terms. Through Planck-suppressed interactions with the moduli, sparticles then obtain the soft SUSY breaking masses as

$$
m_{\text {soft }} \sim \frac{m_{3 / 2}^{2}}{(\text { modulus mass })},
$$

where $m_{3 / 2}$ denotes the gravitino mass. Hence, if stabilized with mass much larger than $4 \pi^{2} m_{3 / 2}$, the modulus does not play an important role in generating the soft SUSY breaking terms because they always receive anomaly-mediated SUSY breaking contribution, $m_{\text {soft }} \sim$ $m_{3 / 2} / 4 \pi^{2}$, in supergravity [38-40].

The stabilization of Kähler moduli can be examined within the effective theory after integrating out the dilation and complex structure moduli as they are much heavier than the gravitino and Kähler moduli [44, 45]. Let us consider a simple case with a single Kähler modulus $X .{ }^{1}$ It is straightforward to generalize to a case with multi Kähler moduli. The Kähler potential takes the no-scale form at the leading order in the $\alpha^{\prime}$ (the string tension) and the string loop expansions,

$$
K_{0}=-3 \ln \left(X+X^{*}\right) .
$$

It should be noticed that the theory possesses the axionic shift symmetry,

$$
\mathrm{U}(1)_{X}: \quad \operatorname{Im}(X) \rightarrow \operatorname{Im}(X)+\text { constant. }
$$

This shift symmetry can be explicitly broken at non-perturbative level. For example, hidden gaugino condensation or stringy instanton effects induce non-perturbative terms in

\footnotetext{
${ }^{1}$ With an abuse of notation, we shall use the same symbol to denote both a chiral superfield and its scalar component, unless noted otherwise.
} 
superpotential. Including the non-perturbative corrections, the modulus superpotential is given by

$$
W_{0}=\omega_{0}+W_{\mathrm{np}}(X)
$$

where the constant term $\omega_{0}$ is originated from background fluxes, which is assumed to be much smaller than order unity in the Planck unit so as to get the soft SUSY breaking scale much lower than the Planck scale. We also note that the constant term explicitly breaks $\mathrm{U}(1)_{R}$. The above superpotential stabilizes the modulus at a SUSY AdS minimum satisfying

$$
\partial_{X} W_{0}+\left(\partial_{X} K_{0}\right) W_{0}=0
$$

Here $\partial_{X}$ means a partial derivative with respect to the scalar field $X$, i.e., $\partial_{X} \equiv \frac{\partial}{\partial X}$. Then the modulus mass is determined by the curvature around the minimum as

$$
m_{X} \simeq\left|\left\langle\frac{\partial_{X} K_{0}}{\partial_{X}^{2} K_{0}} \frac{\partial_{X}^{2} W_{\mathrm{np}}}{\partial_{X} W_{\mathrm{np}}}\right\rangle m_{3 / 2}\right|
$$

where the gravitino mass is given by $m_{3 / 2}=\left\langle e^{K / 2} W\right\rangle \sim \omega_{0}$, and we have used $\partial_{X} K_{0}=$ $\partial_{X^{*}} K_{0}$ and the SUSY condition (2.5). After adding a sequestered uplifting potential $V_{\text {lift }} \propto$ $e^{2 K_{0} / 3}$ to cancel the negative cosmological constant, a small vacuum shift is induced. This vacuum shift results in a nonzero modulus $F$-term,

$$
\left\langle F^{X}\right\rangle \simeq-\frac{2 m_{3 / 2}^{*}}{\left\langle\partial_{X}^{2} W_{\mathrm{np}} / \partial_{X} W_{\mathrm{np}}\right\rangle},
$$

which is order of $\left|m_{3 / 2}\right|^{2} / m_{X}$.

Let us continue to examine how the SUSY breaking is mediated to the visible sector. The soft SUSY breaking terms receive contributions from both modulus mediation and anomaly mediation [44-47],

$$
-\mathcal{L}_{\mathrm{soft}}=m_{i}^{2}\left|\phi_{i}\right|^{2}+\left(\frac{1}{2} M_{a} \lambda_{a} \lambda_{a}+A_{i j k} y_{i j k} \phi_{i} \phi_{j} \phi_{k}+\text { h.c. }\right)
$$

where $\phi_{i}$ denotes the scalar component of the visible sector chiral superfield $\Phi_{i}, \lambda_{a}$ is the gaugino, and $y_{i j k}$ is the Yukawa coupling for the superpotential term $\Phi_{i} \Phi_{j} \Phi_{k}$. Here $i, j, k$ are the indices of matter fields, and $a=1,2,3$ corresponds to the $\mathrm{U}(1)_{Y}, \mathrm{SU}(2)_{L}, \mathrm{SU}(3)_{C}$ gauge group, respectively. The mass of gaugino is obtained as

$$
\begin{aligned}
M_{a}(\Lambda) & =-\left\langle F^{X} \partial_{X} \ln g_{a}^{2}(\Lambda)\right\rangle+\frac{b_{a} g_{a}^{2}(\Lambda)}{16 \pi^{2}} m_{3 / 2}^{*} \\
& =\left\langle\frac{F^{X}}{X+X^{*}}\right\rangle+\frac{b_{a} g_{a}^{2}(\Lambda)}{16 \pi^{2}} m_{3 / 2}^{*},
\end{aligned}
$$

when the gauge kinetic function is

$$
f_{a}(\Lambda)=k_{a} X
$$


at the cut-off scale $\Lambda$, which we assume to be the GUT scale. $k_{a}$ is a positive constant. Here we note that the gauge kinetic function is allowed to have only a term which is linear to $X$ (except for constant term) due to the axionic shift symmetry, i.e., eq. (2.3). The gauge coupling reads $g_{a}^{2}=1 /\left\langle\operatorname{Re}\left(f_{a}\right)\right\rangle$, and the beta-function coefficients are given by $b_{a}=(33 / 5,1,-3)$ in the MSSM. On the other hand, $m_{i}$ and $A_{i j k}$ depend on the anomalous dimension $\gamma_{i}$ and the modular weight $n_{i}$ of $\phi_{i}[48,49]$ that determines the modulus dependence of the matter wave function $Z_{i}{ }^{2}$ For instance, the $A$-parameter reads

$$
\begin{aligned}
A_{i j k}(\Lambda) & =\left\langle F^{X} \partial_{X} \ln \left(Z_{i} Z_{j} Z_{k}\right)\right\rangle-\frac{\gamma_{i}(\Lambda)+\gamma_{j}(\Lambda)+\gamma_{k}(\Lambda)}{16 \pi^{2}} m_{3 / 2}^{*} \\
& =\left(n_{i}+n_{j}+n_{k}\right)\left\langle\frac{F^{X}}{X+X^{*}}\right\rangle-\frac{\gamma_{i}(\Lambda)+\gamma_{j}(\Lambda)+\gamma_{k}(\Lambda)}{16 \pi^{2}} m_{3 / 2}^{*},
\end{aligned}
$$

where $n_{i}$ is a rational number of order unity, and it can have various values in the KKLTtype moduli stabilization with anomalous $\mathrm{U}(1)$ gauge symmetry [50]. Low energy values of the soft SUSY breaking parameters are determined by the renormalization group running, and extra gauge-charged matters if they exist at an intermediate scale [51-54].

\subsection{CP violation in the moduli sector}

As will be shown in the next section, in order to generate sufficient baryon asymmetry from modulus decays, the phase of the combination $A_{i j k} M_{\tilde{g}}^{*}$ should be sizable, i.e.,

$$
\arg \left(A_{i j k} M_{\tilde{g}}^{*}\right) \neq 0,
$$

where $M_{\tilde{g}}$ is the gluino mass, and hereafter $A_{i j k}$ denotes the soft trilinear parameter associated with baryon-number violating superpotential terms, which will be presented soon.

In the KKLT scenario, moduli and anomaly mediations are comparable to each other, and the relative phase between the modulus $F$-term and the gravitino mass is not rotated away in the presence of two or more non-perturbative superpotential terms. This indeed makes it as a natural framework to implement the baryogenesis in the modulus-dominated Universe. From the relations (2.9) and (2.11), the phase is naively estimated to be

$$
\arg \left(A_{i j k} M_{\tilde{g}}^{*}\right) \sim \frac{\arg \left(m_{3 / 2}\left\langle F^{X}\right\rangle\right)}{\alpha+\alpha^{-1}},
$$

omitting an order unity coefficient which depends on the renormalization group running and extra gauge-charged matter fields. The parameter $\alpha$ represents the ratio between moduli and anomaly mediations,

$$
\alpha \equiv\left|\left\langle\frac{F^{X}}{X+X^{*}}\right\rangle^{-1} \frac{m_{3 / 2}}{4 \pi^{2}}\right| \approx \frac{m_{X}}{8 \pi^{2} m_{3 / 2}},
$$

where we have used that the modulus $F$-term is given by eq. (2.7). The original KKLT scenario gives $\alpha \simeq \ln \left(M_{P} / m_{3 / 2}\right) / 4 \pi^{2}=\mathcal{O}(1)$. It is obvious that the phase is suppressed if one of the mediation mechanisms dominates over the other. On the other hand, the

\footnotetext{
${ }^{2}$ The expression of the scalar soft SUSY breaking terms can be found, for instance, in ref. [47].
} 
approximate symmetry, $\mathrm{U}(1)_{R}$ and $\mathrm{U}(1)_{X}$, indicates that $W_{\mathrm{np}}$ should include at least two non-perturbative terms to get nonzero $\arg \left(m_{3 / 2}\left\langle F^{X}\right\rangle\right)$. This is understood as follows. Suppose that there is only a single non-perturbative term. One can then remove the $\mathrm{CP}$ phases in the superpotential by redefining $\omega_{0}$ and $X$. However, it is not generically possible to rotate away the $\mathrm{CP}$ phase(s) of additional non-perturbative term(s). That is why at least two non-perturbative terms are needed to get a CP phase. ${ }^{3}$

Now let us estimate the CP phase more quantitatively. For this purpose, we consider the superpotential,

$$
W_{0}=\omega_{0}-A e^{-a X}-B e^{-b X},
$$

with $a \neq b$ and $a \sim b \sim 4 \pi^{2}$, where $A$ and $B$ are order unity complex constants. Here the approximate $\mathrm{U}(1)_{R}$ and $\mathrm{U}(1)_{X}$ (explicitly broken by the constant and non-perturbative terms) allow us to make $\omega_{0}$ and $A$ be real and positive numbers without loss of generality, which is the convention we will take hereafter. This explains why $X$ is fixed at a CPconserving minimum with mass

$$
m_{X} \simeq 2 m_{3 / 2} \ln \left(M_{P} / m_{3 / 2}\right),
$$

in the absence of the last term in eq. (2.15), as was considered in the original KKLT mechanism. Here we have used $m_{3 / 2} \sim \omega_{0}$ and $A \sim 1$. Let us now turn on the $B e^{-b X}$ term with $\operatorname{Im}(B) \neq 0$. Then the SUSY condition reads

$$
a A e^{-a X}+b B e^{-b X} \simeq \frac{3 \omega_{0}}{X+X^{*}},
$$

neglecting small terms suppressed by $1 / a\left(X+X^{*}\right)$ or $1 / b\left(X+X^{*}\right)$. Since the righthand side is a real number, $\operatorname{Im}(B) \neq 0$ shifts the minimum to a CP-violating one, i.e., $\langle\operatorname{Im}(X)\rangle \neq 0$. Using the facts that the modulus $F$-term is generated according to the relation (2.7) after uplifting, and that the modulus is fixed at $a\langle\operatorname{Re}(X)\rangle \simeq \ln \left(M_{P} / m_{3 / 2}\right)$, we find that $\arg \left(m_{3 / 2}\left\langle F^{X}\right\rangle\right)$ is determined by

$$
\arg \left(m_{3 / 2}\left\langle F^{X}\right\rangle\right) \simeq-\arg \left\langle\frac{\partial_{X}^{2} W_{\mathrm{np}}}{\partial_{X} W_{\mathrm{np}}}\right\rangle
$$

where the superpotential (2.15) gives

$$
\frac{\partial_{X}^{2} W_{\mathrm{np}}}{\partial_{X} W_{\mathrm{np}}}=-a-(b-a) \frac{b B e^{-b X}}{a A e^{-a X}+b B e^{-b X}} .
$$

Therefore, the CP phase can be sizable if $a A e^{-a X}$ and $b B e^{-b X}$ are comparable to each other in size. ${ }^{4}$ Under the assumption that $\left|b B e^{-b X}\right| \lesssim\left|a A e^{-a X}\right|$, the vacuum shift induced

\footnotetext{
${ }^{3}$ The mirage unification pattern of gaugino masses $[46,47]$ is violated in our baryogenesis scenario where the $\mathrm{CP}$ violation results from a relative phase between the modulus F-term and the gravitino mass.

${ }^{4}$ One may consider a racetrack-type model [55] where the modulus is stabilized mainly by the competition between two non-perturbative terms in the superpotential while the constant piece is negligibly small. However, in this case, two approximate symmetries $\mathrm{U}(1)_{R}$ and $\mathrm{U}(1)_{X}$ fix the modulus near a CP preserving minimum. Furthermore, the modulus becomes much heavier than $m_{3 / 2} \ln \left(M_{P} / m_{3 / 2}\right)$, thereby suppressing the modulus mediation. See the relation (2.13).
} 
by $\operatorname{Im}(B)$ leads to

$$
\arg \left(m_{3 / 2}\left\langle F^{X}\right\rangle\right) \approx \frac{b(b-a)}{a^{2}}\left(\frac{m_{3 / 2}}{M_{P}}\right)^{\frac{b-a}{a}} \frac{\operatorname{Im}(B)}{A},
$$

for $b$ similar to but larger than $a$. Here $a / b$ is a rational number if the non-perturbative terms arise from hidden gaugino condensation. For instance, the phase is around 0.05-0.1 $m_{3 / 2} \sim 10^{4}-10^{8} \mathrm{GeV}$ in the model with $(b-a) / a=1 / 10$ for $A \sim 0.1$ and $\operatorname{Im}(B) \sim 1$.

Let us close this section by briefly mentioning the SUSY breaking scale. We are interested in the case where the $\mathrm{CP}$ violation in the moduli sector gives rise to a sizable $\arg \left(A_{i j k} M_{\tilde{g}}^{*}\right)$ to implement the baryogenesis. Thus, to avoid the SUSY CP problems, the MSSM sparticles should be heavier than $1 \mathrm{TeV}$ unless there is a cancellation among sparticle contributions in the amplitudes of physical processes. We will quantitatively discuss the constraint on the $\mathrm{CP}$ phase later. Heavy sparticles around over $\mathrm{TeV}$ scale may indicate a little hierarchy problem regarding the Higgs boson mass. However, to put it another way, such a large sparticle (mainly stop) mass is one of possible explanations for the $126 \mathrm{GeV}$ Higgs boson discovered at the LHC [56, 57].

\section{Baryogenesis}

In this section we will discuss the generation of baryon asymmetry by modulus decay. During inflation the modulus is likely deviated from the true vacuum in the low energy, due to the deformation of the potential through gravitational interactions with the inflaton. After inflation ends, it starts coherent oscillation when the Hubble parameter becomes comparable to $m_{X}$ with a large initial displacement of the order of the Planck scale, and then soon dominates the energy density of the Universe. Eventually the modulus field decays to lighter particles. The modulus decay releases a huge amount of entropy, which would dilute any (harmful) relic of the early Universe, and reheats the Universe at the temperature $T_{X}$ given by

$$
T_{X} \simeq\left(\frac{90}{\pi^{2} g_{*}\left(T_{X}\right)}\right)^{1 / 4} \sqrt{\Gamma_{X} M_{P}}
$$

where $g_{*}$ is the relativistic degrees of freedom, and $\Gamma_{X}$ is the total decay width of the modulus field. Although the late modulus decay might washout pre-existing baryon asymmetry as well, we will show that sufficient baryon asymmetry is generated by the modulus decay followed by the gluino decay if the R-parity is violated. Here we will also discuss the experimental consequences of this baryogenesis scenario.

\subsection{Baryon asymmetry}

The modulus decays into the MSSM particles through its couplings to the visible sector. For the case that the gauge couplings are determined by $\langle X\rangle$, the modulus $X$ dominantly decays into gauge boson pairs, and gaugino pairs through the interactions (written in twocomponent notation) [32-35],

$$
\mathcal{L}_{X}=\frac{c_{g}^{a}}{4}\left(\delta X_{r} G_{\mu \nu}^{a} G^{a \mu \nu}-\delta X_{i} G_{\mu \nu}^{a} \tilde{G}^{a \mu \nu}\right)-\frac{c_{\lambda}^{a}}{4}\left(\delta X_{r} \lambda_{a} \lambda_{a}-i \delta X_{i} \lambda_{a} \lambda_{a}+\text { h.c. }\right),
$$


with $\delta X_{r}+i \delta X_{i} \equiv\left\langle 2 \partial_{X}^{2} K_{0}\right\rangle^{1 / 2}(X-\langle X\rangle)$ being the canonically normalized fluctuation about the vacuum. Here $G_{\mu \nu}^{a}$ is the field strength of gauge field, and the modulus couplings are given by

$$
\begin{aligned}
c_{g}^{a} & =\sqrt{2} \frac{\partial_{X} \ln g_{a}^{2}}{\sqrt{\partial_{X}^{2} K_{0}}}, \\
c_{\lambda}^{a} & =c_{g}^{a}\left(1+\mathcal{O}\left(\frac{m_{3 / 2}}{m_{X}}\right)\right) m_{X},
\end{aligned}
$$

where the modulus coupling to gauginos has been derived by using the relation

$$
\partial_{X} F^{X^{*}}=-e^{K_{0} / 2} \frac{\partial_{X}^{2} W_{0}}{\partial_{X}^{2} K_{0}}\left(1+\mathcal{O}\left(\frac{m_{3 / 2}}{m_{X}}\right)\right),
$$

evaluated at the vacuum. Then the decay rates to a gauge boson pair and a gaugino pair are given by

$$
\begin{aligned}
& \Gamma\left(X \rightarrow A^{a} A^{a}\right)=\frac{1}{96 \pi} \frac{m_{X}^{3}}{M_{P}^{2}}, \\
& \Gamma\left(X \rightarrow \lambda_{a} \lambda_{a}\right)=\frac{1}{96 \pi} \frac{m_{X}^{3}}{M_{P}^{2}}\left[1-\frac{4 M_{a}^{2}}{m_{X}^{2}}\right]\left[1-\frac{5 M_{a}^{2}}{2 m_{X}^{2}}\right],
\end{aligned}
$$

respectively, for both the real and imaginary components of $X$. Here $A^{a}$ denotes the gauge boson, neglecting its mass, and we have used the gauge kinetic function $f_{a}=k_{a} X$. Under the assumption that modulus decays into hidden sector particles are suppressed, the total decay rate of the modulus is simply written as

$$
\Gamma_{X} \simeq \frac{1}{4 \pi} \frac{m_{X}^{3}}{M_{P}^{2}}
$$

neglecting the gaugino mass in the final state. Then, from eqs. (3.1) and (3.7), the reheating temperature is estimated as

$$
T_{X} \simeq 98 \mathrm{GeV}\left(\frac{g_{*}}{106.75}\right)^{-1 / 4}\left(\frac{m_{X}}{10^{8} \mathrm{GeV}}\right)^{3 / 2}
$$

Since the decay rate is Planck-suppressed, the reheating temperature is usually lower than the typical SUSY breaking scale, which means that the produced sparticles are out of equilibrium. This is an essential ingredient, i.e., for the Sakharov condition (ii), for the baryogenesis via subsequent gluino and squark decays. It is also important that the branching fraction into a gluino pair is given by

$$
\operatorname{Br}(X \rightarrow \tilde{g} \tilde{g})=\frac{\Gamma(X \rightarrow \tilde{g} \tilde{g})}{\Gamma_{X}} \simeq \frac{1}{3} .
$$

Thus gluinos, whose decay is the source of the baryon asymmetry, are abundantly produced by modulus decay. 

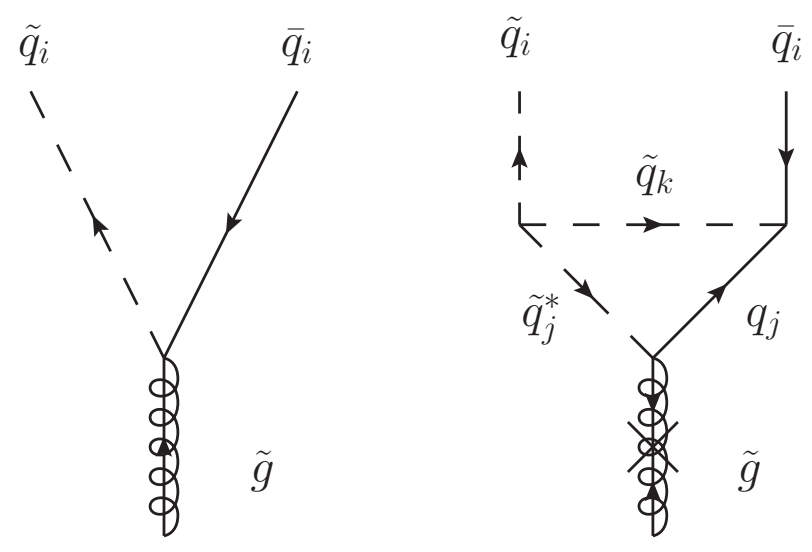

Figure 1. Gluino decay diagrams to a quark and a squark. The cross in the gluino line represents a chirality flip.

For the baryogenesis we introduce the baryon-number violating renormalizable operators in addition to the MSSM superpotential $W_{\mathrm{MSSM}}{ }^{5}$

$$
W_{\mathrm{vis}}=W_{\mathrm{MSSM}}+\frac{1}{2} \lambda_{i j k} U_{i}^{c} D_{j}^{c} D_{k}^{c}
$$

where $U_{i}^{c}$ and $D_{j}^{c}$ are the $\mathrm{SU}(2)_{L}$ singlet up-type and down-type quarks, respectively, and $i, j, k$ are flavor indices while color indices are implicit. The other renormalizable lepton number violating operators can be forbidden by generalized lepton parities or discrete $\mathrm{R}$ symmetries [59]. From the superpotential we obtain the following Lagrangian,

$$
\begin{aligned}
\mathcal{L}_{R_{p}}= & -\lambda_{i j k}\left(\tilde{d}_{k}^{c}\left(\bar{u}_{i} P_{L} d_{j}^{c}\right)+\tilde{u}_{i}^{c}\left(\bar{d}_{j} P_{L} d_{k}^{c}\right)\right)-\lambda_{i j k}^{*}\left(\tilde{d}_{k}^{c *}\left(\bar{d}_{j}^{c} P_{R} u_{i}\right)+\tilde{u}_{i}^{c *}\left(\bar{d}_{k}^{c} P_{R} d_{j}\right)\right) \\
& +\frac{1}{2}\left(A_{i j k} \lambda_{i j k} \tilde{u}_{i}^{c} \tilde{d}_{j}^{c} \tilde{d}_{k}^{c}+A_{i j k}^{*} \lambda_{i j k} \tilde{u}_{i}^{c *} \tilde{d}_{j}^{c *} \tilde{d}_{k}^{c *}\right) .
\end{aligned}
$$

Here the tilde denotes the scalar superpartner, and $P_{R / L}$ are projection operators defined as $P_{R / L}=\left(1 \pm \gamma_{5}\right) / 2$ (plus for $P_{R}$ and minus for $P_{L}$ ). The first line in the right-hand side includes SUSY couplings, while the second line is the soft SUSY breaking trilinear terms, which correspond to the second term in eq. (2.8).

The gluino mainly decays to quark and squark through the tree-level diagram in figure 1 . The decay rate for $\tilde{g} \rightarrow \tilde{q}_{i} \bar{q}_{i}$ reads

$$
\Gamma\left(\tilde{g} \rightarrow \tilde{q}_{i} \bar{q}_{i}\right)=\frac{\alpha_{s}}{8}\left|M_{\tilde{g}}\right|\left(1-r_{i}\right)^{2},
$$

where $r_{i}=m_{\tilde{q}_{i}}^{2} /\left|M_{\tilde{g}}\right|^{2}$, neglecting the quark mass. Here $\alpha_{s}=g_{3}^{2} / 4 \pi$ is the strong coupling constant, and $m_{\tilde{q}_{i}}$ denotes the mass of squark in the final state. The total decay rate of

\footnotetext{
${ }^{5}$ Instead, we can consider other R-parity violating operators which break lepton number. In such a case, nonzero lepton number would be generated in a similar manner as is illustrated in the following discussion. Then the lepton number is converted to baryon number via the sphaleron process. See ref. [58].
} 
the gluino is given by the sum of all possible final sates of quark and squark,

$$
\Gamma_{\tilde{g}}=\sum_{i}\left[\Gamma\left(\tilde{g} \rightarrow \tilde{q}_{i} \bar{q}_{i}\right)+\Gamma\left(\tilde{g} \rightarrow \tilde{q}_{i}^{*} q_{i}\right)\right]
$$

On the other hand, an asymmetry between $\Gamma\left(\tilde{g} \rightarrow \tilde{q}_{i} \bar{q}_{i}\right)$ and $\Gamma\left(\tilde{g} \rightarrow \tilde{q}_{i}^{*} q_{i}\right)$ may be induced at the loop level. If there exists another decay mode to $\tilde{q}_{j}^{*} q_{j}$, the interference between tree and loop diagrams (figure 1) generates nonzero asymmetry via the baryon-number violating operator given in eq. (3.11);

$$
\begin{aligned}
\Delta \Gamma\left(\tilde{g} \rightarrow \tilde{q}_{R i} \bar{q}_{R i}\right) & \equiv \Gamma\left(\tilde{g} \rightarrow \tilde{q}_{R i} \bar{q}_{R i}\right)-\Gamma\left(\tilde{g} \rightarrow \tilde{q}_{R i}^{*} q_{R i}\right) \\
& =\sum_{j, k} c_{i j} \frac{\alpha_{s}}{32 \pi} \frac{\left|\lambda_{i j k}\right|^{2} \operatorname{Im}\left(A_{i j k} M_{\tilde{g}}^{*}\right)}{\left|M_{\tilde{g}}\right|} f\left(r_{i}, r_{j}, r_{k}\right),
\end{aligned}
$$

where

$$
f\left(r_{i}, r_{j}, r_{k}\right)=\left(1-r_{i}\right)\left(1-r_{j}\right)-r_{k} \ln \left(1+r_{k}^{-1}\left(1-r_{i}\right)\left(1-r_{j}\right)\right) .
$$

Here the constant $c_{i j}$ has a nonzero value only for the process kinematically allowed: $c_{i j}=2$ if the intermediate and final states (i.e., $\tilde{q}_{j}^{*} q_{j}$ and $\tilde{q}_{i} \bar{q}_{i}$ ) are both down-type (s)quarks, and $c_{i j}=1$ otherwise. In the above, we have ignored left-right mixing in the squark sector for simplicity. It is straightforward to take into account the left-right mixing. The function $f\left(r_{i}, r_{j}, r_{k}\right)$ behaves as $f\left(r_{i}, r_{j}, r_{k}\right) \simeq 1$ when $r_{i, j, k} \ll 1$. On the other hand, it is possible that $r_{k}$ is greater than one. If $r_{k}>1$ (and $r_{i, j} \ll 1$ ), then it is suppressed as $f\left(r_{i}, r_{j}, r_{k}\right) \simeq 1 / 2 r_{k}$. This behavior is expected because the loop diagram in figure 1 should vanish in the limit $m_{\tilde{q}_{k}} \rightarrow \infty .^{6}$

Now we discuss net baryon number generated by the gluino decay. First we write the (s)quark number which is generated by the decay of a single gluino as

$$
\Delta n_{\tilde{q}_{i}}=-\Delta n_{q_{i}}=\frac{\Delta \Gamma\left(\tilde{g} \rightarrow \tilde{q}_{R i} \bar{q}_{R i}\right)}{\Gamma_{\tilde{g}}} .
$$

Even though it is zero just after the gluino decay, nonzero baryon number is generated by subsequent $\Delta B \neq 0$ squark decay processes. Possible decay modes of the squark are $\Delta B=1$ processes such as $\tilde{q}_{i} \rightarrow \bar{q}_{j} \bar{q}_{k}$, and $\Delta B=0$ processes such as $\tilde{q} \rightarrow \tilde{\chi}^{0} q$ with $\tilde{\chi}^{0}$ being a neutralino. Here the $\Delta B=1$ process also includes the case where $\tilde{q} \rightarrow \tilde{q}^{\prime} W / Z$ and subsequently $\tilde{q}^{\prime}$ decays to quark pairs via the baryon-number violating operator. Eventually the net baryon number generated by a single gluino is obtained as

$$
\begin{aligned}
\epsilon_{B} & \equiv \sum_{i} \Delta n_{\tilde{q}_{i}}\left[\operatorname{Br}^{\tilde{q}_{i}} \times\left(-\frac{2}{3}\right)+\left(1-\mathrm{Br}^{\tilde{q}_{i}}\right) \times \frac{1}{3}\right]+\sum_{i} \Delta n_{q_{i}} \times \frac{1}{3} \\
& =-\sum_{i} \Delta n_{\tilde{q}_{i}} \mathrm{Br}^{\tilde{q}_{i}} .
\end{aligned}
$$

\footnotetext{
${ }^{6}$ This point was not discussed in ref. [22].
} 
Here $\mathrm{Br}^{\tilde{q}_{i}}$ is the branching fraction for the $\Delta B=1$ decay process, which can be the dominant mode when the $\lambda_{i j k}$ is order unity, or it is unity when $\tilde{q}_{i}$ is the lightest sparticle. Then, the baryon number density from a gluino decay is

$$
\begin{aligned}
n_{B} & =n_{\tilde{g}} \epsilon_{B} \\
& =2 n_{X} \operatorname{Br}(X \rightarrow \tilde{g} \tilde{g}) \epsilon_{B} \\
& \simeq \frac{2}{3} n_{X} \epsilon_{B},
\end{aligned}
$$

where $n_{\tilde{g}}$ and $n_{X}$ are the number density of gluino and the modulus, respectively, and we have used eq. (3.9) at the last step. Under the assumption that all the energy density of the modulus turns into radiation at its decay, i.e., $\rho_{X}=m_{X} n_{X} \simeq \rho_{R}$ ( $\rho_{R}$ is the energy density of radiation), the yield of baryon asymmetry is obtained as

$$
\frac{n_{B}}{s}=\frac{3 T_{X}}{4 m_{X}} 2 \operatorname{Br}(X \rightarrow \tilde{g} \tilde{g}) \epsilon_{B}
$$

Let us estimate the baryon asymmetry in this baryogenesis scenario. For the estimation of the asymmetry, we assume that only $\lambda_{332}$ is nonzero for simplicity. ${ }^{7}$ The total decay width of the gluino is then given by

$$
\Gamma_{\tilde{g}} \simeq 4 n_{f} \Gamma\left(\tilde{g} \rightarrow \tilde{q}_{i} \bar{q}_{i}\right) \simeq \frac{\alpha_{s} n_{f}}{2}\left|M_{\tilde{g}}\right| .
$$

Here a factor four counts CP conjugate, and left-handed and right-handed fields, and $n_{f}$ is the number of quark flavors to which gluino can decay. We have taken the limit $r_{i} \ll 1$ for the kinematically allowed decay for simplicity. In the same limit and taking $n_{f}=6$, then the asymmetry is given by

$$
\begin{aligned}
\Delta \Gamma_{\tilde{g}} & =\sum_{i} \Delta \Gamma\left(\tilde{g} \rightarrow \tilde{q}_{R i} \bar{q}_{R i}\right) \\
& \simeq \frac{\alpha_{s}}{4 \pi} \frac{\left|\lambda_{332}\right|^{2} \operatorname{Im}\left(A_{332} M_{\tilde{g}}^{*}\right)}{\left|M_{\tilde{g}}\right|},
\end{aligned}
$$

which leads to ${ }^{8}$

$$
\frac{\Delta \Gamma_{\tilde{g}}}{\Gamma_{\tilde{g}}} \simeq \frac{1}{12 \pi} \frac{\left|\lambda_{332}\right|^{2} \operatorname{Im}\left(A_{332} M_{\tilde{g}}^{*}\right)}{\left|M_{\tilde{g}}\right|^{2}}
$$

This is the typical scale of asymmetric parameter when the gluino is much heavier than the squarks. Furthermore taking $\mathrm{Br}^{\tilde{t}, \tilde{b}, \tilde{s}}=\mathrm{Br}^{\tilde{q}}$, we get

$$
\epsilon_{B} \simeq 1.3 \times 10^{-3}\left(\frac{\left|\lambda_{332}\right|^{2} \operatorname{Im}\left(A_{332} M_{\tilde{g}}^{*}\right) /\left|M_{\tilde{g}}\right|^{2}}{-0.1}\right)\left(\frac{\mathrm{Br}^{\tilde{q}}}{0.5}\right),
$$

\footnotetext{
${ }^{7}$ Actually the case where only $\lambda_{332}$ is sizable is realistic in the phenomenological point of view. One of well-motivated scenarios is the minimal flavor violation [60].

${ }^{8}$ This expression has a different factor compared to the result given in ref. [22]. In ref. [22], it is $-\frac{1}{16 \pi}$ instead of $\frac{1}{12 \pi}$.
} 


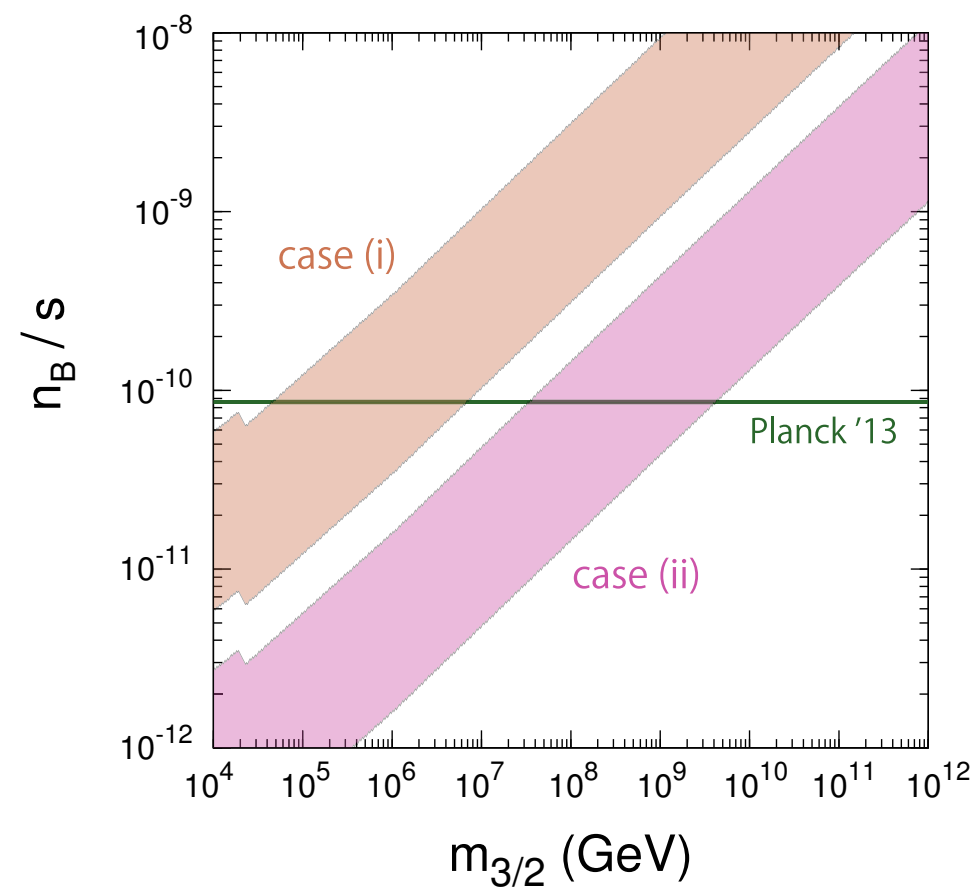

Figure 2. Baryon asymmetry generated by modulus decays as a function of the gravitino mass for cases where $(i) m_{\tilde{t}, \tilde{b}, \tilde{s}}=m_{\mathrm{soft}}, \operatorname{Br}^{\tilde{t}, \tilde{b}, \tilde{s}}=0.5$ and $(i i) m_{\tilde{t}, \tilde{b}}=m_{\mathrm{soft}}, \operatorname{Br}^{\tilde{t}, \tilde{b}}=0.5$, where $m_{\text {soft }}=$ $m_{3 / 2} / 4 \pi^{2}$. For both cases, the gluino mass is taken as $M_{\tilde{g}}=3 m_{\mathrm{soft}}$, and the other squark masses are taken to be $m_{\tilde{q}}=6 m_{\text {soft }}$. For the modulus mass, we take $m_{X}=2 m_{3 / 2} \ln \left(M_{P} / m_{3 / 2}\right)$ following eq. (2.16). Upper and lower bands correspond to the baryon asymmetry in case $(i)$ and $(i i)$, respectively. For both cases, the $\mathrm{CP}$ phase is in the region, $0.01 \leq-\left|\lambda_{332}\right|^{2}\left(\operatorname{Im}\left(A_{332} M_{\tilde{g}}^{*}\right) /\left|M_{\tilde{g}}\right|^{2}\right) \leq$ 0.1. The observed value given in eq. (1.1) is depicted as "Planck '13".

which results in

$$
\frac{n_{B}}{s} \simeq 4.9 \times 10^{-10}\left(\frac{g_{*}}{106.75}\right)^{-1 / 4}\left(\frac{m_{X}}{10^{8} \mathrm{GeV}}\right)^{1 / 2}\left(\frac{\epsilon_{B}}{10^{-3}}\right)
$$

Therefore, the observed baryon asymmetry of the Universe can be obtained with $\mathcal{O}(0.1)$ $\mathrm{CP}$ phase and $\mathcal{O}(1)$ baryon-number violating coupling if the modulus $X$ has a mass around $10^{6}-10^{8} \mathrm{GeV}$. In the later numerical calculation, we also take into account the effect of sphaleron processes. Namely, we replace $n_{B} / s$ as $(28 / 79) \times n_{B} / s$ when $T_{X} \gtrsim 4 \pi m_{W} / g_{2}^{2}$, where $m_{W}$ is the $W$ boson mass.

\subsection{Numerical result}

In this subsection we give the numerical results. Since we are considering the KKLT moduli stabilization where the modulus and anomaly mediations give comparable contributions to the soft SUSY breaking terms, the typical mass scale for the sparticles is given by

$$
m_{\mathrm{soft}}=\frac{m_{3 / 2}}{4 \pi^{2}}
$$


The precise sparticle mass spectrum is model-dependent, i.e., it relies on the details of moduli stabilization and moduli couplings to the visible sector, renormalization group flow from the cut-off scale, and presence or absence of intermediate scale gauge-charged matters. In the following analysis we parametrize the relevant sparticle masses by $m_{\text {soft }}$, and use the relation eq. (2.16) for the modulus mass. For simplicity, we take only $\lambda_{332}$ to be nonzero. Let us consider the following two examples:

$$
\begin{aligned}
\text { (i) } \quad M_{\tilde{g}}=3 m_{\mathrm{soft}}, \quad m_{\tilde{t}, \tilde{b}, \tilde{s}}=m_{\mathrm{soft}}, \quad m_{\tilde{q} \neq \tilde{f}, \tilde{b}, \tilde{s}}=6 m_{\mathrm{soft}}, \quad \operatorname{Br}^{\tilde{t}, \tilde{b}, \tilde{s}}=0.5, \\
\text { (ii) } M_{\tilde{g}}=3 m_{\mathrm{soft}}, \quad m_{\tilde{t}, \tilde{b}}=m_{\mathrm{soft}}, \quad m_{\tilde{q} \neq \tilde{t}, \tilde{b}}=6 m_{\mathrm{soft}}, \quad \operatorname{Br}^{\tilde{t}, \tilde{b}}=0.5 .
\end{aligned}
$$

For both cases we take the effective $\mathrm{CP}$ violation parameter to be

$$
0.01 \leq-\left|\lambda_{332}\right|^{2} \frac{\operatorname{Im}\left(A_{332} M_{\tilde{g}}^{*}\right)}{\left|M_{\tilde{g}}\right|^{2}} \leq 0.1
$$

where we have taken into account that the KKLT moduli stabilization leads to CP violation according to eq. (2.20) and assumed $\left|\lambda_{332}\right| \sim \mathcal{O}(1)$. The observed baryon asymmetry given in eq. (1.1) is drawn in the plot. The baryon asymmetry in the case (ii) is expected to be suppressed by $M_{\tilde{g}}^{2} / m_{\tilde{s}}^{2}$ as we mentioned in the previous subsection. We show it as an reference of one of the possible SUSY mass spectra.

Figure 2 shows $n_{B} / s$ as a function of $m_{3 / 2}$. The baryon asymmetry from the modulus decay is shown by the upper band for the case $(i)$, and by the lower one for the case $(i i)$. We have checked the out-of-equilibrium condition for the gluino and the squark is fulfilled in a wide range of the parameter space, such as $m_{3 / 2} \lesssim 10^{14} \mathrm{GeV}$. One can see that, in the case $(i)$, the correct amount of baryon asymmetry is generated when the gravitino mass is around $10^{5}-10^{7} \mathrm{GeV}$, which corresponds to $m_{\text {soft }} \sim 10^{3}-10^{5} \mathrm{GeV}$. This is consistent with a rough estimation given in eq. (3.24). On the other hand, in the case (ii), the suppression by the strange squark mass makes the baryon asymmetry smaller compared to the previous case by around an order of magnitude. ${ }^{9}$ As a consequence, a larger gravitino mass is required to account for the baryon asymmetry of the Universe. The figure shows that the gravitino mass should lie in the range about $10^{7}-10^{10} \mathrm{GeV}$.

The gravitino can be produced by the modulus decay with a branching fraction of $\mathcal{O}(0.01)$. The produced gravitino acts as radiation until it decays because it is originally produced relativistically. Thus it never dominates the Universe, and it does not cause significant entropy production. In addition, the gravitino decay does not destroy the successful $\mathrm{BBN}$ if $m_{3 / 2} \gtrsim 10 \mathrm{TeV}$, which is satisfied in the parameter range of our interest. Therefore, the asymmetry generated by modulus (and subsequent sparticle) decays is not diluted. It is also important to note that the moduli-induced gravitino problem [32-35] is avoided in the presence of R-parity violating operators because the lightest sparticle is not stable anymore.

\footnotetext{
${ }^{9}$ The suppression factor can be estimated as follows. Since the gluino cannot decay to strange (s)quark, $\Gamma_{\tilde{g}}$ becomes $(2 / 3) \times \Gamma_{\tilde{g}}$ compared to the case $(i)$, and $\Delta \Gamma_{\tilde{g}}$ becomes $(1 / 4)\left(M_{\tilde{g}}^{2} / 2 m_{\tilde{s}}^{2}\right) \times \Delta \Gamma_{\tilde{g}}$. This gives a suppression factor $3 / 63$, which is consistent with the numerical result given in figure 2 .
} 
Finally we comment on constraints from other experiments. A large CP phase coming from $\arg \left(A_{i j k} M_{\tilde{g}}^{*}\right)$ may induce sizable electric dipole moments (EDMs) of neutron and electron. The current experimental bounds are given by [61, 62],

$$
\begin{array}{ll}
d_{n} \leq 2.9 \times 10^{-26} e \mathrm{~cm} & (90 \% \text { C.L. }), \\
d_{e} \leq 8.7 \times 10^{-29} e \mathrm{~cm} & (90 \% \text { C.L. }),
\end{array}
$$

for neutron and electron EDMs, respectively. We have estimated the neutron and electron EDM based on refs. [63-65] (see also refs. [66, 67] and ref. [68]), and found that both constraints give a similar bound as

$$
\operatorname{Im}\left(A_{i j k} M_{\tilde{g}}^{*}\right) /\left|M_{\tilde{g}}\right|^{2} \lesssim 0.1 \times\left(\frac{m_{\text {soft }}}{1 \mathrm{TeV}}\right)^{2} .
$$

Here we have taken the universal soft SUSY breaking mass, and neglected the CP phase from $\mu / B \mu$ term [47]. Therefore the current bound allows $\mathcal{O}(0.1) \mathrm{CP}$ phase even if sparticles are as low as around $\mathrm{TeV}$, which confirms the parametrization in the numerical calculation, i.e., eq. (3.26). If the $\mathrm{CP}$ phase from $\mu / B \mu$ term is included, the bound may become more stringent by a factor, depending on the model. In any case, it is interesting since the parameter space we are interested in may be probed in the ongoing or future measurements of the EDMs.

The baryon-number violating operators possibly induce neutron-antineutron $(n-\bar{n})$ oscillation or dinucleon decay. Those experiments constrain the coupling in light flavors, such as $u d \tilde{s}$ (in two-component notation). However, even if $\lambda_{112}$ is negligibly small, sizable $\lambda_{332}$ induces $u d \tilde{s}$-type coupling at one loop. For instance, $\lambda_{332}=1$ gives a value of $\mathcal{O}\left(10^{-7}\right)$ for the light flavor coupling [69]. Thus it may constrain our baryogenesis scenario. SuperKamiokande experiment gives constraint on the time scale of $n-\bar{n}$ oscillation and dinucleon decay in ${ }^{16} \mathrm{O}[70,71]$,

$$
\begin{array}{ll}
\tau_{n-\bar{n}} \geq 2.4 \times 10^{8} \mathrm{sec} & (90 \% \text { C.L. }), \\
\tau\left(p p \rightarrow K^{+} K^{-}\right) \geq 1.7 \times 10^{32} \text { yr } & (90 \% \text { C.L. })
\end{array}
$$

We have estimated the time scale of $n-\bar{n}$ oscillation and the decay rate of $p p \rightarrow K^{+} K^{-}$ based on refs. $[69,72]$, then we found the experimental bounds above give rise to

$$
\begin{aligned}
& \left|\lambda_{112}\right| \lesssim 4.4 \times 10^{-3}\left(\frac{m_{\text {soft }}}{1 \mathrm{TeV}}\right)^{5 / 6}\left(\frac{250 \mathrm{MeV}}{\tilde{\Lambda}}\right) \\
& \left|\lambda_{112}\right| \lesssim 3.2 \times 10^{-7}\left(\frac{m_{\text {soft }}}{1 \mathrm{TeV}}\right)^{5 / 2}\left(\frac{250 \mathrm{MeV}}{\tilde{\Lambda}}\right)^{5 / 2},
\end{aligned}
$$

respectively. Here we have set $M_{\tilde{g}}=m_{\tilde{q}}=m_{\text {soft }}$ for simplicity. $\tilde{\Lambda}$ is the hadronic scale used in the evaluation of the hadronic matrix element, which has theoretical uncertainty. As it is seen, the dinucleon decay experiment gives more stringent constraint than $n-\bar{n}$ oscillation. Especially the bound from dinucleon decay is interesting. Recall that the numerical result implies that $m_{\tilde{q}} \sim \mathcal{O}(1) \mathrm{TeV}$ with $\lambda_{332} \sim \mathcal{O}$ (1) leads to the right abundance of baryon (i.e., case(i)). This means that a part of the parameter region where our baryogenesis works can be probed in the current or future experiments. In addition, the squark with a mass of $\mathcal{O}(1) \mathrm{TeV}$ may be discovered at the collider experiments, such as the LHC or the ILC. 


\section{Other scenarios}

So far we have discussed baryogenesis by the modulus decay. As it has been shown, the KKLT scenario has important ingredients for the baryogenesis, i.e., suppressed interactions of the moduli with the visible sector, a large enough CP phase and a large branching fraction to gluinos in the modulus decay. Other than the KKLT scenario, there are several possible scenarios which possess (some of) the ingredients stated above. In this section we will discuss the gravitino- and the saxion-induced baryogenesis.

\subsection{Gravitino-induced baryogenesis}

The baryogenesis by the gravitino decay was originally discussed by Cline and Raby in ref. [22]. In their work relatively low SUSY breaking scale, which is around several hundred $\mathrm{GeV}$, was studied. Recently ref. [58] pursued the gravitino-induced leptogenesis using lepton number violating operators. Here we revisit the original case by Cline and Raby, also considering high scale SUSY breaking region.

The gravitino is produced effectively at a high temperature. If the reheating temperature after inflation is high enough, the gravitino is copiously generated, and eventually dominates the Universe. In addition, the gravitino can also be produced by the inflaton decay [26-31]. In the typical mass spectra given in eq. (3.25), the gravitino decays to the MSSM particles. The total decay rate is given by

$$
\Gamma_{3 / 2}=\sum_{i}\left[\Gamma\left(\psi_{\mu} \rightarrow \tilde{\phi}_{i} \phi_{i}\right)+\Gamma\left(\psi_{\mu} \rightarrow \overline{\tilde{\phi}}_{i} \phi_{i}^{*}\right)\right]+\sum_{a} \Gamma\left(\psi_{\mu} \rightarrow \lambda_{a} A_{a}\right),
$$

where $\psi_{\mu}$ is the gravitino, $\tilde{\phi}_{i}$ is the fermionic superpartner of scalar $\phi_{i}$ and ${ }^{10}$

$$
\begin{aligned}
& \Gamma\left(\psi_{\mu} \rightarrow \tilde{\phi}_{i} \phi_{i}\right)=\frac{1}{192 \pi} \frac{m_{3 / 2}^{3}}{M_{P}^{2}}\left[1-\frac{m_{\phi}^{2}}{m_{3 / 2}^{2}}\right]^{4}, \\
& \Gamma\left(\psi_{\mu} \rightarrow \lambda_{a} A_{a}\right)=\frac{1}{32 \pi} \frac{m_{3 / 2}^{3}}{M_{P}^{2}}\left[1-\frac{M_{a}^{2}}{m_{3 / 2}^{2}}\right]^{3}\left[1+\frac{M_{a}^{2}}{3 m_{3 / 2}^{2}}\right] .
\end{aligned}
$$

Here we have neglected gauge boson masses. Since the decay rates are Planck suppressed as in moduli decay, the reheating temperature is typically the same as eq. (3.8). Now let us suppose that the gravitino decays to all MSSM particles. Then taking the large gravitino mass limit, the total decay width is given by

$$
\Gamma_{3 / 2} \simeq \frac{1}{32 \pi}\left(\frac{1}{3} N+N_{g}\right) \frac{m_{3 / 2}^{3}}{M_{P}^{2}}
$$

where $N=49$ is the number of chiral superfields (neglecting right-handed neutrinos) and $N_{g}=12$ is the number of gauginos in the MSSM. Then the reheating temperature is

\footnotetext{
${ }^{10}$ We found a typo for $\Gamma\left(\psi_{\mu} \rightarrow \tilde{\phi}_{i} \phi_{i}\right)$ given in ref. [73]. We thank T. Moroi for confirming this point. For $\Gamma\left(\psi_{\mu} \rightarrow \lambda_{a} A_{a}\right)$, we got consistent result with ref. [73], which is four times smaller than the result given in ref. [22].
} 
estimated as

$$
T_{3 / 2} \simeq 1.9 \times 10^{2} \mathrm{GeV}\left(\frac{g_{*}}{106.75}\right)^{-1 / 4}\left(\frac{m_{3 / 2}}{10^{8} \mathrm{GeV}}\right)^{3 / 2} .
$$

In the gravitino decay a fair amount of gluinos is produced if it is kinematically allowed. The branching ratio of the decay to gluino and gluon, under the assumption above, is then

$$
\operatorname{Br}\left(\psi_{\mu} \rightarrow \tilde{g} g\right)=\frac{\Gamma\left(\psi_{\mu} \rightarrow \tilde{g} g\right)}{\Gamma_{3 / 2}} \simeq \frac{8}{N / 3+N_{g}}=\frac{24}{85} .
$$

This large branching fraction is important for the baryogenesis. Net baryon asymmetry from the gluino decay is then expressed as

$$
\left.\frac{n_{B}}{s}\right|_{\text {gluino }}=\frac{3 T_{3 / 2}}{4 m_{3 / 2}} \operatorname{Br}\left(\psi_{\mu} \rightarrow \tilde{g} g\right) \epsilon_{B} .
$$

Using eqs. (4.5) and (4.6), $n_{B} / s$ is estimated as

$$
\left.\frac{n_{B}}{s}\right|_{\text {gluino }} \simeq 3.9 \times 10^{-10}\left(\frac{g_{*}}{106.75}\right)^{-1 / 4}\left(\frac{m_{3 / 2}}{10^{8} \mathrm{GeV}}\right)^{1 / 2}\left(\frac{\epsilon_{B}}{10^{-3}}\right) .
$$

On the other hand, the decay modes to quark and squark are also important. In the mass spectrum mentioned above, the branching fraction is

$$
\operatorname{Br}\left(\psi_{\mu} \rightarrow \tilde{q}_{R} \bar{q}_{R}\right)=\frac{\sum_{i}\left[\Gamma\left(\psi_{\mu} \rightarrow \tilde{q}_{R i} \bar{q}_{R i}\right)+\Gamma\left(\psi_{\mu} \rightarrow \tilde{q}_{R i}^{*} q_{R i}\right)\right]}{\Gamma_{3 / 2}} \simeq \frac{3 n_{f}}{N / 3+N_{g}}=\frac{9 n_{f}}{85},
$$

where $n_{f}$ is the number of quark flavors which can be produced by the gravitino decay. As it is seen, the branching fraction can be comparable to $\operatorname{Br}\left(\psi_{\mu} \rightarrow \tilde{g} g\right)$. Note that this decay mode has the asymmetry between its $\mathrm{CP}$ conjugate and itself, which is given by a similar diagram depicted in figure 1 just by replacing the gluino with the gravitino. Therefore if physical CP phase $\operatorname{Im}\left(A_{i j k} m_{3 / 2}^{*}\right)$ is nonzero, then baryon asymmetry is generated. As a result of straightforward calculation, we get

$$
\Delta \Gamma\left(\psi_{\mu} \rightarrow \tilde{q}_{R i} \bar{q}_{R i}\right)=\sum_{j, k} \frac{1}{256 \pi^{2}} \frac{\left|\lambda_{i j k}\right|^{2} \operatorname{Im}\left(A_{i j k} m_{3 / 2}^{*}\right)\left|m_{3 / 2}\right|}{M_{P}^{2}} g\left(r_{i}, r_{j}, r_{k}\right),
$$

where

$$
\begin{aligned}
g\left(r_{i}, r_{j}, r_{k}\right)= & \left(1-r_{i}\right)\left(1-r_{j}\right)\left[\left(1-r_{i}\right)\left(1-r_{j}\right)+6 r_{k}\right] \\
& -2 r_{k}\left[2\left(1-r_{i}\right)\left(1-r_{j}\right)+3 r_{k}\right] \ln \left[1+\frac{\left(1-r_{i}\right)\left(1-r_{j}\right)}{r_{k}}\right] .
\end{aligned}
$$

Here $r_{i}=m_{\tilde{q}_{i}}^{2} /\left|m_{3 / 2}\right|^{2}$. Similarly to $f\left(r_{i}, r_{j}, r_{k}\right), g\left(r_{i}, r_{j}, r_{k}\right)$ approaches to unity when $r_{i, j, k} \ll 1$, while it is suppressed as $g\left(r_{i}, r_{j}, r_{k}\right) \simeq 1 / 2 r_{k}$ when $r_{k}>1$ and $r_{i, j} \ll 1$ as expected. Then net baryon number directly generated by the gravitino decay is obtained as

$$
\epsilon_{B}^{\prime} \equiv-\sum_{i} \frac{\Delta \Gamma\left(\psi_{\mu} \rightarrow \tilde{q}_{R i} \bar{q}_{R i}\right)}{\Gamma_{3 / 2}} \operatorname{Br}^{\tilde{q}_{i}}
$$


Now, let us consider only nonzero $\lambda_{332}$ as in the section 3.1. In the large gravitino mass limit, we get ${ }^{11}$

$$
\sum_{i} \Delta \Gamma\left(\psi_{\mu} \rightarrow \tilde{q}_{R i} \bar{q}_{R i}\right) \simeq \frac{1}{32 \pi^{2}} \frac{\left|\lambda_{332}\right|^{2} \operatorname{Im}\left(A_{332} m_{3 / 2}^{*}\right)\left|m_{3 / 2}\right|}{M_{P}^{2}},
$$

which gives rise to

$$
\sum_{i} \frac{\Delta \Gamma\left(\psi_{\mu} \rightarrow \tilde{q}_{R i} \bar{q}_{R i}\right)}{\Gamma_{3 / 2}} \simeq \frac{3}{260 \pi} \frac{\left|\lambda_{332}\right|^{2} \operatorname{Im}\left(A_{332} m_{3 / 2}^{*}\right)}{\left|m_{3 / 2}\right|^{2}} .
$$

Taking $\mathrm{Br}^{\tilde{t}, \tilde{b}, \tilde{s}}=\mathrm{Br}^{\tilde{q}}$, net baryon number generated due to this process is given by

$$
\epsilon_{B}^{\prime} \simeq 5.6 \times 10^{-4}\left(\frac{\left|\lambda_{332}\right|^{2} \operatorname{Im}\left(A_{332} m_{3 / 2}^{*}\right) /\left|m_{3 / 2}\right|^{2}}{-0.1}\right)\left(\frac{\mathrm{Br}^{\tilde{q}}}{0.5}\right) .
$$

Then baryon asymmetry directly generated by the gravitino decay to quark and squark is

$$
\begin{aligned}
\left.\frac{n_{B}}{s}\right|_{\text {squark }} & =\frac{3 T_{3 / 2}}{4 m_{3 / 2}} \epsilon_{B}^{\prime} \\
& \simeq 8.3 \times 10^{-10}\left(\frac{g_{*}}{106.75}\right)^{-1 / 4}\left(\frac{m_{3 / 2}}{10^{8} \mathrm{GeV}}\right)^{1 / 2}\left(\frac{\epsilon_{B}^{\prime}}{6 \times 10^{-4}}\right) .
\end{aligned}
$$

Therefore, it is seen that the baryon number which is resulted in the processes $\psi_{\mu} \rightarrow \tilde{q}_{R} \bar{q}_{R}$, $\tilde{q}_{R}^{*} q_{R}$ has the same order as gluino-mediated one.

Finally let us compare those results with ref. [22]. In their work, they took the maximum value for $\mathrm{CP}$ phase which is allowed by constraints from neutron EDM experiment at that time. $\left(d_{n} \leq 10^{-26} e \mathrm{~cm}\right.$ was used there.) We have estimated the CP phase by following the way they described in their paper. Then taking $\mathrm{Br}^{\tilde{q}_{i}}=1$ and $\left|\lambda_{332}\right|=4 \pi \times 0.1$ as they did, we get

$$
\begin{aligned}
\frac{n_{B}}{s} & =\left.\frac{n_{B}}{s}\right|_{\text {gluino }}+\left.\frac{n_{B}}{s}\right|_{\text {squark }} \\
& \simeq 1.6 \times 10^{-10}\left(\frac{g_{*}}{10.75}\right)^{-1 / 4}\left(\frac{m_{3 / 2}}{2 \mathrm{TeV}}\right)^{1 / 2} .
\end{aligned}
$$

This is almost consistent with ref. [22] up to $\mathcal{O}(1)$ factor.

\subsection{Saxion-induced baryogenesis}

The Peccei-Quinn (PQ) mechanism is a plausible solution to the strong CP problem, and it predicts a pseudo Nambu-Goldstone boson, the axion [74-80], which acquires a small mass predominantly from the QCD anomaly. The axion is stable on cosmological time scale, thus it is a good candidate for dark matter. In the supersymmetric extension of the PQ mechanism, its scalar partner, called saxion, remains relatively light as it acquires a mass only from the SUSY breaking effects. In fact, it is known that the saxion tends to dominate

\footnotetext{
${ }^{11}$ In ref. [22] a factor $\frac{3}{4} \frac{1}{32 \pi^{2}}$ is given instead of $\frac{1}{32 \pi^{2}}$.
} 
the energy density of the early Universe, and it plays a similar role as the modulus. The advantages of using the saxion as a source of the baryogenesis are two folds. First, it necessarily couples to the QCD gauge sector in order to solve the strong CP problem, and therefore naturally decays into gluons and gluinos. Secondly, the baryogenesis can be more efficient because the decay temperature will be relatively high for the axion decay constant $F_{a}$ is smaller than the GUT scale.

Let us assume that the coherent oscillation of the saxion dominates the energy density of the Universe. The amount of baryon asymmetry generated by the saxion decay depends on the reheating temperature $T_{s}$ at the saxion decay as well as the branching fraction for the saxion decay into a gluino pair. In particular, the latter depends on the saxion coupling to axion,

$$
\frac{\xi}{F_{a}} s(\partial a)^{2}
$$

where $s$ and $a$ are the saxion and axion, respectively, and $\xi$ is a model-dependent numerical coefficient. In order to have a sizable branching fraction into gluino pair, and also to avoid the overproduction of axionic dark radiation, $\xi$ must be highly suppressed below unity since the saxion coupling to the QCD gauge sector is one-loop suppressed for a given axion decay constant $F_{a} \cdot{ }^{12}$ As the precise value of $\xi$ depends on how the PQ scalars are stabilized, let us see the axion models in some detail.

The axion models can be categorized according to whether the PQ symmetry is realized linearly or non-linearly. For the former case, which is sometimes dubbed a field-theoretic axion model, the axion decay constant $F_{a}$ ranges from an intermediate scale up to the GUT scale. The saxion coupling with axion depends on the stabilization mechanism. In a model with a single PQ scalar, $\xi$ is of order unity. One way to suppress $\xi$ is to consider a model with two PQ scalars $S_{1}$ and $S_{2}$, which have an opposite PQ charge each other, and a $\mathrm{U}(1)_{\mathrm{PQ}}$ singlet $\Sigma$,

$$
W_{\mathrm{PQ}}=\lambda \Sigma\left(S_{1} S_{2}-\mu^{2}\right),
$$

so that the PQ symmetry is broken along the $F$-flat direction $S_{1} S_{2}=\mu^{2}$, which is lifted by SUSY breaking effects. In this model, the axion decay constant is given by $\mu$. If $S_{1}$ and $S_{2}$ obtain the soft scalar masses of a similar size, then $\xi$ is suppressed as $\xi^{2} \sim \Delta m_{S}^{2} / m_{S}^{2}$, where $m_{S}^{2}$ is the typical size of their soft masses and $\Delta m_{S}^{2}$ is the mass splitting between them [82].

The latter case, which is called a string axion model, on the other hand, corresponds to the case where the axionic shift symmetry of some moduli remains unbroken (except for the QCD anomaly). The axion decay constant is then typically around $M_{P} / 8 \pi^{2}$ unless the Kähler metric of the saxion is hierarchically smaller than unity at the vacuum. Thus, in this case, one needs to assume a small initial misalignment of the axion in order for the axion relic energy density not to overclose the Universe. On the other hand, the saxion

\footnotetext{
${ }^{12}$ The branching fraction into the axions can also be suppressed by introducing a coupling of the saxion to the Higgs fields [81] or the right-handed neutrinos [82]. The axionic dark radiation has been extensively studied in e.g., refs. [81-87].
} 
coupling to axion is typically suppressed as $\xi \sim 1 / 8 \pi^{2}$, and thus the branching fraction of the saxion decay into gluino pair is naturally sizable and is comparable to that into the axions [85]. This can be understood by noting that the coupling to the QCD gauge sector arises at tree level in this case.

We note that the KKLT mechanism provides a natural framework to implement the saxion-induced baryogenesis both for the field-theoretic and the string axion models. First, the CP phase is obtained in the same way as explained in section 2. Note that the Fterm in the axion multiplet does not induce a new CP phase in the MSSM soft terms because the soft terms generating a saxion potential depend on the moduli $F$-terms and the gravitino mass. Besides, the suppression for $\xi$ can be also implemented in both models with the KKLT scenario. For the axion model described in eq. (4.19), let us assume that the Yukawa couplings of $S_{1}$ and $S_{2}$ are small, and the PQ scalars $S_{1}$ and $S_{2}$ have the same modulus dependence, i.e., the same modular weight. Since the symmetry under interchanging between $S_{1}$ and $S_{2}$ is a good symmetry, then one can naturally achieve $\Delta m_{S}^{2} \ll m_{S}^{2}$, suppressing the saxion coupling to the axion. On the other hand, in the string axion model, the QCD axion can arise from a Kähler modulus in the KKLT scenario with multiple Kähler moduli, if the axionic shift symmetry for the Kähler modulus is not broken explicitly. [88, 89]. This modulus is stabilized with a mass of about $\sqrt{2} m_{3 / 2}$ through Kähler mixing with other moduli, which are stabilized by the non-perturbative superpotential as in the original KKLT.

In either field-theoretic axion model or string axion model, the modulus $X$ may dominate the Universe before the saxion-dominated Universe. This is because the saxion is much lighter than the modulus. Although the modulus decay generates the baryon asymmetry as we have described, the asymmetry is washed out by the saxion decay later. However, the saxion decay can generate baryon asymmetry.

Now let us estimate the baryon asymmetry produced by the saxion. For simplicity we consider the case where the axion production from the saxion decays is negligible, and the saxion dominantly decays to a gluon pair and a gluino pair. Including the decay into the axions does not affect our results as long as $|\xi| \lesssim 1 / 8 \pi^{2}$.

The saxion couplings to gluon and gluino are given (in two-component notation) by

$$
\mathcal{L}_{s}=\frac{\alpha_{s}}{8 \pi} \frac{s}{F_{a}} G_{\mu \nu} G^{\mu \nu}-\frac{\alpha_{s}}{8 \pi} \frac{m_{s} s}{F_{a}}(\kappa \tilde{g} \tilde{g}+\text { h.c. }),
$$

where $G_{\mu \nu}$ is the gluon field strength and $m_{s}$ is the saxion mass, and the order unity constant $\kappa$ is determined by how the saxion is stabilized. The above couplings mediate the saxion decay into a gluon pair and a gluino pair with

$$
\begin{aligned}
\Gamma(s \rightarrow g g) & =\frac{\alpha_{s}}{32 \pi^{3}} \frac{m_{s}^{3}}{F_{a}^{2}}, \\
\Gamma(s \rightarrow \tilde{g} \tilde{g}) & =\frac{\alpha_{s}}{32 \pi^{3}} \frac{m_{s}^{3}}{F_{a}^{2}}\left[1-\frac{4 M_{\tilde{g}}^{2}}{m_{s}^{2}}\right]\left[|\kappa|^{2}\left(1-\frac{M_{\tilde{g}}^{2}}{2 m_{s}^{2}}\right)-2 \operatorname{Re}\left(\kappa^{2}\right) \frac{M_{\tilde{g}}^{2}}{m_{s}^{2}}\right] .
\end{aligned}
$$

Then the total decay rate of the saxion is given as

$$
\Gamma_{s} \simeq \frac{\alpha_{s}}{32 \pi^{3}} \frac{m_{s}^{3}}{F_{a}^{2}}\left(1+|\kappa|^{2}\right),
$$


neglecting the gluino mass in the final state. Consequently the branching ratio for the process $s \rightarrow \tilde{g} \tilde{g}$ is obtained as

$$
\operatorname{Br}(s \rightarrow \tilde{g} \tilde{g}) \simeq \frac{|\kappa|^{2}}{1+|\kappa|^{2}}
$$

On the contrary, $\operatorname{Br}(s \rightarrow \tilde{g} \tilde{g}) \simeq\left(1+m_{s}^{2} / 8 M_{\tilde{g}}^{2}\right)^{-1}$ was given in ref. [23]. This result indicates a chiral suppression due to the gluino mass since $\operatorname{Br}(s \rightarrow \tilde{g} \tilde{g}) \rightarrow 0$ when $M_{\tilde{g}} \rightarrow 0$. As it is seen, however, there is no such chiral suppression in eq. (4.24), which is already pointed out by ref. [37]. Since $|\kappa|$ is order unity, the saxion can dominantly decay into a gluino pair. This makes the baryogenesis by the saxion decay more efficient than originally considered in ref. [23].

After the gluino production, the same story follows as in the modulus case. In the string axion models, the reheating temperature is similar to the modulus case, while it can be higher in field-theoretic axion models if the axion decay constant is smaller than the GUT scale. The reheating temperature is given by

$$
T_{s} \simeq 0.73 \mathrm{GeV}\left(\frac{g_{*}}{10.75}\right)^{-1 / 4}\left(\frac{10^{16} \mathrm{GeV}}{F_{a}}\right)\left(\frac{m_{s}}{10^{5} \mathrm{GeV}}\right)^{3 / 2}
$$

where we have taken $\kappa=1$. The resultant asymmetric yield is estimated by

$$
\begin{aligned}
\frac{n_{B}}{s} & =\frac{3 T_{s}}{4 m_{s}} 2 \operatorname{Br}(s \rightarrow \tilde{g} \tilde{g}) \epsilon_{B} \\
& \simeq 5.4 \times 10^{-10}\left(\frac{g_{*}}{10.75}\right)^{-1 / 4}\left(\frac{m_{s}}{10^{5} \mathrm{GeV}}\right)^{1 / 2}\left(\frac{10^{16} \mathrm{GeV}}{F_{a}}\right)\left(\frac{\operatorname{Br}(s \rightarrow \tilde{g} \tilde{g})}{1 / 2}\right)\left(\frac{\epsilon_{B}}{10^{-3}}\right) .
\end{aligned}
$$

Thus the right amount of baryon asymmetry can be generated for the saxion mass around $10^{3}-10^{5} \mathrm{GeV}$. On top of that, as mentioned above, the axion produced by the misalignment mechanism contributes to the cold dark matter. This fact is especially important because the lightest sparticle is no longer stable due to the R-parity violation.

\section{Conclusions and Discussion}

In this paper we have studied a baryogenesis induced by late-decaying moduli, and examined its implications for the moduli stabilization and the SUSY breaking scale. If the branching fraction of the modulus into a gluino pair is sizable, the right amount of baryon asymmetry can be generated through $\mathrm{CP}$ violating decay of gluino into quark and squark followed by baryon-number violating squark decays. We have shown that a natural framework realizing the baryogenesis is provided by the KKLT-type moduli stabilization since sufficient CP violation is obtained from mixed modulus-anomaly mediated SUSY breaking in the presence of two or more non-perturbative terms in the modulus superpotential. Successful baryogenesis is possible for the gravitino mass around $10^{5}-10^{7} \mathrm{GeV}$ or equivalently the soft SUSY breaking mass $m_{\text {soft }} \sim 10^{3}-10^{5} \mathrm{GeV}$ (or heavier for a suppressed CP phase or effective CP violation parameter). Such low SUSY breaking scale can be probed directly 
at the collider experiments, dinucleon decay search, and electric dipole moments of neutron and electron. We also found that similar baryogenesis works successfully in other scenarios where the saxion or the gravitino dominates the Universe.

Lastly let us discuss baryogenesis in a couple of other moduli stabilization and SUSY breaking scenarios. We have mainly focused on the mixed modulus-anomaly mediation so far, assuming a sequestered uplifting. If the uplifting is not sequestered, sfermion masses are generically heavier than gaugino masses, then the gluino decay into quark and squark will be kinematically forbidden. Even in such case, however, the gravitino produced by the modulus decay will be able to generate baryon asymmetry.

The large volume scenario [90] can also lead to CP violating soft terms if one takes the same form of the superpotential for a small 4-cycle modulus as in Eq. (2.15). However, one cannot simply apply the moduli-induced baryogenesis discussed in the present paper to the large volume scenario because the overall volume modulus, which has a small mass compared to other moduli, does not appear in the visible gauge kinetic function and thus weakly couples to gluino. In addition, the modulus decay into a pair of gravitinos is kinematically forbidden and the decay into Higgsinos is suppressed due to the approximate no-scale structure even in the presence of the Giudice-Masiero term [84]. Those fact indicate much smaller amount of gluino produced by modulus decay, which suppress the resultant baryon number.

Regarding the moduli stabilization, we note that there is a general tension between moduli stabilization by non-perturbative effects and chirality [91]. To be specific, (a combination of) the moduli $X_{L}$ appearing in the gauge kinetic function of the visible gauge sector may not be stabilized by instantonic exponential terms. The saxion in the string axion model corresponds to this case, and as discussed before, it can be stabilized by another way $[88,89]$ and the saxion-induced baryogenesis works. Alternatively, we may consider a modulus field which is stabilized a la KKLT, while not appearing in the SM gauge kinetic function. Such modulus field can still decay into gauge bosons and gauginos through the kinetic mixing with $X_{L}$. Thus the baryon asymmetry will be generated by the subsequent gluino decay, although it may be diluted by the decay of lighter moduli $X_{L}$ to some extent.

Similar baryogenesis can be also realized in gravity mediation. In the gravity-mediated SUSY breaking, the MSSM gaugino masses are generated by Planck-scale suppressed interactions with an elementary gauge singlet field called the Polonyi field. Since it is a singlet under any symmetry of the theory, there is no special point in its field space. Therefore, during inflation, the Polonyi field is generically deviated from the true minimum, as the effective potential for the Polonyi field during inflation is deformed by its gravitational couplings with the inflaton. After inflation, the Polonyi starts to oscillate about the minimum with an amplitude of order the Planck scale, and soon dominates the energy density of the Universe. The Polonyi field will decay into the MSSM gauge sector, and thus the baryogenesis by the Polonyi decay will be possible. In particular, a sizable A-term as well as large CP phases are naturally generated in the gravity mediation, which nicely fits with the current scenario. The resultant baryon asymmetry is similar to (3.24) if the modulus mass is replaced with the Polonyi mass, which is order of the gravitino mass. 


\section{Acknowledgments}

This work is supported by Grant-in-Aid for Scientific Research on Innovative Areas (No. 24111702, No. 21111006, and No. 23104008) [FT], Scientific Research (A) (No. 22244030 and No. 21244033) [FT], and JSPS Grant-in-Aid for Young Scientists (B) (No. 24740135) [FT], Inoue Foundation for Science, and by World Premier International Center Initiative (WPI Program), MEXT, Japan [FT]. This publication is partly funded by the Gordon and Betty Moore Foundation through Grant GBMF \#776 to the Caltech Moore Center for Theoretical Cosmology and Physics. The work is also supported by the U.S. Department of Energy under Contract No. DE-FG02-92ER40701 [KI].

Open Access. This article is distributed under the terms of the Creative Commons Attribution License (CC-BY 4.0), which permits any use, distribution and reproduction in any medium, provided the original author(s) and source are credited.

\section{References}

[1] Planck collaboration, P.A.R. Ade et al., Planck 2013 results. XVI. Cosmological parameters, arXiv:1303.5076 [INSPIRE].

[2] A.D. Sakharov, Violation of CP Invariance, c Asymmetry and Baryon Asymmetry of the Universe, Pisma Zh. Eksp. Teor. Fiz. 5 (1967) 32 [JETP Lett. 5 (1967) 24] [Sov. Phys. Usp. 34 (1991) 392] [Usp. Fiz. Nauk 161 (1991) 61] [INSPIRE].

[3] A.H. Guth, The Inflationary Universe: A Possible Solution to the Horizon and Flatness Problems, Phys. Rev. D 23 (1981) 347 [INSPIRE].

[4] A.A. Starobinsky, A New Type of Isotropic Cosmological Models Without Singularity, Phys. Lett. B 91 (1980) 99 [INSPIRE].

[5] K. Sato, First Order Phase Transition of a Vacuum and Expansion of the Universe, Mon. Not. Roy. Astron. Soc. 195 (1981) 467 [inSPIRE].

[6] A.D. Linde, A New Inflationary Universe Scenario: A Possible Solution of the Horizon, Flatness, Homogeneity, Isotropy and Primordial Monopole Problems, Phys. Lett. B 108 (1982) 389 [INSPIRE].

[7] A. Albrecht and P.J. Steinhardt, Cosmology for Grand Unified Theories with Radiatively Induced Symmetry Breaking, Phys. Rev. Lett. 48 (1982) 1220 [INSPIRE].

[8] M. Fukugita and T. Yanagida, Baryogenesis Without Grand Unification, Phys. Lett. B 174 (1986) 45 [INSPIRE].

[9] I. Affleck and M. Dine, A New Mechanism for Baryogenesis, Nucl. Phys. B 249 (1985) 361 [INSPIRE].

[10] M. Dine, L. Randall and S.D. Thomas, Baryogenesis from flat directions of the supersymmetric standard model, Nucl. Phys. B 458 (1996) 291 [hep-ph/9507453] [INSPIRE].

[11] E.D. Stewart, M. Kawasaki and T. Yanagida, Affleck-Dine baryogenesis after thermal inflation, Phys. Rev. D 54 (1996) 6032 [hep-ph/9603324] [INSPIRE].

[12] T. Asaka and M. Kawasaki, Cosmological moduli problem and thermal inflation models, Phys. Rev. D 60 (1999) 123509 [hep-ph/9905467] [INSPIRE]. 
[13] S. Kasuya, M. Kawasaki and F. Takahashi, On the moduli problem and baryogenesis in gauge mediated SUSY breaking models, Phys. Rev. D 65 (2002) 063509 [hep-ph/0108171] [INSPIRE].

[14] D.-h. Jeong, K. Kadota, W.-I. Park and E.D. Stewart, Modular cosmology, thermal inflation, baryogenesis and predictions for particle accelerators, JHEP 11 (2004) 046 [hep-ph/0406136] [INSPIRE].

[15] M. Kawasaki and K. Nakayama, Baryon Asymmetry in Heavy Moduli Scenario, Phys. Rev. D 76 (2007) 043502 [arXiv:0705.0079] [INSPIRE].

[16] T. Higaki, K. Kamada and F. Takahashi, Higgs, Moduli Problem, Baryogenesis and Large Volume Compactifications, JHEP 09 (2012) 043 [arXiv: 1207.2771] [INSPIRE].

[17] M. Kawasaki, K. Kohri and N. Sugiyama, Cosmological constraints on late time entropy production, Phys. Rev. Lett. 82 (1999) 4168 [astro-ph/9811437] [INSPIRE].

[18] M. Kawasaki, K. Kohri and N. Sugiyama, MeV scale reheating temperature and thermalization of neutrino background, Phys. Rev. D 62 (2000) 023506 [astro-ph/0002127] [INSPIRE].

[19] S. Hannestad, What is the lowest possible reheating temperature?, Phys. Rev. D 70 (2004) 043506 [astro-ph/0403291] [INSPIRE].

[20] K. Ichikawa, M. Kawasaki and F. Takahashi, The Oscillation effects on thermalization of the neutrinos in the Universe with low reheating temperature, Phys. Rev. D 72 (2005) 043522 [astro-ph/0505395] [INSPIRE].

[21] S. Kachru, R. Kallosh, A.D. Linde and S.P. Trivedi, de Sitter vacua in string theory, Phys. Rev. D 68 (2003) 046005 [hep-th/0301240] [INSPIRE].

[22] J.M. Cline and S. Raby, Gravitino induced baryogenesis: A Problem made a virtue, Phys. Rev. D 43 (1991) 1781 [INSPIRE].

[23] S. Mollerach and E. Roulet, Axino induced baryogenesis, Phys. Lett. B 281 (1992) 303 [INSPIRE].

[24] K. Kohri, A. Mazumdar and N. Sahu, Inflation, baryogenesis and gravitino dark matter at ultra low reheat temperatures, Phys. Rev. D 80 (2009) 103504 [arXiv:0905.1625] [INSPIRE].

[25] C. Cheung and K. Ishiwata, Baryogenesis with Higher Dimension Operators, Phys. Rev. D 88 (2013) 017901 [arXiv: 1304.0468] [INSPIRE].

[26] M. Kawasaki, F. Takahashi and T.T. Yanagida, Gravitino overproduction in inflaton decay, Phys. Lett. B 638 (2006) 8 [hep-ph/0603265] [InSPIRE].

[27] M. Kawasaki, F. Takahashi and T.T. Yanagida, The Gravitino-overproduction problem in inflationary universe, Phys. Rev. D 74 (2006) 043519 [hep-ph/0605297] [INSPIRE].

[28] T. Asaka, S. Nakamura and M. Yamaguchi, Gravitinos from heavy scalar decay, Phys. Rev. D 74 (2006) 023520 [hep-ph/0604132] [INSPIRE].

[29] M. Endo, M. Kawasaki, F. Takahashi and T. Yanagida, Inflaton decay through supergravity effects, Phys. Lett. B 642 (2006) 518 [hep-ph/0607170] [InSPIRE].

[30] M. Endo, F. Takahashi and T.T. Yanagida, Anomaly-induced inflaton decay and gravitino-overproduction problem, Phys. Lett. B 658 (2008) 236 [hep-ph/0701042] [INSPIRE].

[31] M. Endo, F. Takahashi and T.T. Yanagida, Inflaton Decay in Supergravity, Phys. Rev. D 76 (2007) 083509 [arXiv:0706.0986] [INSPIRE]. 
[32] M. Endo, K. Hamaguchi and F. Takahashi, Moduli-induced gravitino problem, Phys. Rev. Lett. 96 (2006) 211301 [hep-ph/0602061] [INSPIRE].

[33] M. Endo, K. Hamaguchi and F. Takahashi, Moduli/Inflaton Mixing with Supersymmetry Breaking Field, Phys. Rev. D 74 (2006) 023531 [hep-ph/0605091] [InSPIRE].

[34] S. Nakamura and M. Yamaguchi, Gravitino production from heavy moduli decay and cosmological moduli problem revived, Phys. Lett. B 638 (2006) 389 [hep-ph/0602081] [INSPIRE].

[35] M. Dine, R. Kitano, A. Morisse and Y. Shirman, Moduli decays and gravitinos, Phys. Rev. D 73 (2006) 123518 [hep-ph/0604140] [INSPIRE].

[36] K.S. Jeong and F. Takahashi, A Gravitino-rich Universe, JHEP 01 (2013) 173 [arXiv: 1210.4077] [INSPIRE].

[37] M. Endo and F. Takahashi, Non-thermal Production of Dark Matter from Late-Decaying Scalar Field at Intermediate Scale, Phys. Rev. D 74 (2006) 063502 [hep-ph/0606075] [INSPIRE].

[38] L. Randall and R. Sundrum, Out of this world supersymmetry breaking, Nucl. Phys. B 557 (1999) 79 [hep-th/9810155] [INSPIRE].

[39] G.F. Giudice, M.A. Luty, H. Murayama and R. Rattazzi, Gaugino mass without singlets, JHEP 12 (1998) 027 [hep-ph/9810442] [INSPIRE].

[40] J.A. Bagger, T. Moroi and E. Poppitz, Anomaly mediation in supergravity theories, JHEP 04 (2000) 009 [hep-th/9911029] [INSPIRE].

[41] V.S. Kaplunovsky and J. Louis, Model independent analysis of soft terms in effective supergravity and in string theory, Phys. Lett. B 306 (1993) 269 [hep-th/9303040] [INSPIRE].

[42] A. Brignole, L.E. Ibáñez and C. Muñoz, Towards a theory of soft terms for the supersymmetric Standard Model, Nucl. Phys. B 422 (1994) 125 [Erratum ibid. B 436 (1995) 747] [hep-ph/9308271] [INSPIRE].

[43] K. Choi, Small SUSY phases in string inspired supergravity, Phys. Rev. Lett. 72 (1994) 1592 [hep-ph/9311352] [INSPIRE].

[44] K. Choi, A. Falkowski, H.P. Nilles, M. Olechowski and S. Pokorski, Stability of flux compactifications and the pattern of supersymmetry breaking, JHEP 11 (2004) 076 [hep-th/0411066] [INSPIRE].

[45] K. Choi, A. Falkowski, H.P. Nilles and M. Olechowski, Soft supersymmetry breaking in KKLT flux compactification, Nucl. Phys. B 718 (2005) 113 [hep-th/0503216] [INSPIRE].

[46] M. Endo, M. Yamaguchi and K. Yoshioka, A Bottom-up approach to moduli dynamics in heavy gravitino scenario: Superpotential, soft terms and sparticle mass spectrum, Phys. Rev. D 72 (2005) 015004 [hep-ph/0504036] [INSPIRE].

[47] K. Choi, K.S. Jeong and K.-i. Okumura, Phenomenology of mixed modulus-anomaly mediation in fluxed string compactifications and brane models, JHEP 09 (2005) 039 [hep-ph/0504037] [INSPIRE].

[48] V.S. Kaplunovsky and J. Louis, Model independent analysis of soft terms in effective supergravity and in string theory, Phys. Lett. B 306 (1993) 269 [hep-th/9303040] [INSPIRE].

[49] A. Brignole, L.E. Ibáñez and C. Muñoz, Towards a theory of soft terms for the supersymmetric Standard Model, Nucl. Phys. B 422 (1994) 125 [Erratum ibid. B 436 (1995) 747-748] [hep-ph/9308271] [INSPIRE]. 
[50] K. Choi and K.S. Jeong, Supersymmetry breaking and moduli stabilization with anomalous U(1) gauge symmetry, JHEP 08 (2006) 007 [hep-th/0605108] [INSPIRE].

[51] L.L. Everett, I.-W. Kim, P. Ouyang and K.M. Zurek, Deflected Mirage Mediation: A Framework for Generalized Supersymmetry Breaking, Phys. Rev. Lett. 101 (2008) 101803 [arXiv:0804.0592] [INSPIRE].

[52] L.L. Everett, I.-W. Kim, P. Ouyang and K.M. Zurek, Moduli Stabilization and Supersymmetry Breaking in Deflected Mirage Mediation, JHEP 08 (2008) 102 [arXiv:0806.2330] [INSPIRE].

[53] K. Choi, K.S. Jeong, S. Nakamura, K.-I. Okumura and M. Yamaguchi, Sparticle masses in deflected mirage mediation, JHEP 04 (2009) 107 [arXiv: 0901.0052] [INSPIRE].

[54] S. Nakamura, K.-i. Okumura and M. Yamaguchi, Axionic Mirage Mediation, Phys. Rev. D 77 (2008) 115027 [arXiv: 0803.3725] [INSPIRE].

[55] N.V. Krasnikov, On Supersymmetry Breaking in Superstring Theories, Phys. Lett. B 193 (1987) 37 [INSPIRE].

[56] ATLAS collaboration, Observation of a new particle in the search for the Standard Model Higgs boson with the ATLAS detector at the LHC, Phys. Lett. B 716 (2012) 1 [arXiv: 1207.7214] [INSPIRE].

[57] CMS collaboration, Observation of a new boson at a mass of $125 \mathrm{GeV}$ with the CMS experiment at the LHC, Phys. Lett. B 716 (2012) 30 [arXiv:1207.7235] [INSPIRE].

[58] L.M. Krauss, A.J. Long and S. Sabharwal, Gravitino Leptogenesis, Phys. Rev. D 89 (2014) 043503 [arXiv: 1309.1454] [INSPIRE].

[59] R. Barbier, C. Berat, M. Besancon, M. Chemtob, A. Deandrea et al., R-parity violating supersymmetry, Phys. Rept. 420 (2005) 1 [hep-ph/0406039] [INSPIRE].

[60] C. Csáki, Y. Grossman and B. Heidenreich, MFV SUSY: A Natural Theory for R-Parity Violation, Phys. Rev. D 85 (2012) 095009 [arXiv:1111.1239] [InSPIRE].

[61] C.A. Baker, D.D. Doyle, P. Geltenbort, K. Green, M.G.D. van der Grinten et al., An Improved experimental limit on the electric dipole moment of the neutron, Phys. Rev. Lett. 97 (2006) 131801 [hep-ex/0602020] [INSPIRE].

[62] ACME collaboration, J. Baron et al., Order of Magnitude Smaller Limit on the Electric Dipole Moment of the Electron, Science (2013) [arXiv:1310.7534] [INSPIRE].

[63] M. Pospelov and A. Ritz, Electric dipole moments as probes of new physics, Annals Phys. 318 (2005) 119 [hep-ph/0504231] [INSPIRE].

[64] J. Hisano, J.Y. Lee, N. Nagata and Y. Shimizu, Reevaluation of Neutron Electric Dipole Moment with QCD Sum Rules, Phys. Rev. D 85 (2012) 114044 [arXiv:1204.2653] [INSPIRE].

[65] J. Hisano, K. Tsumura and M.J.S. Yang, QCD Corrections to Neutron Electric Dipole Moment from Dimension-six Four-Quark Operators, Phys. Lett. B 713 (2012) 473 [arXiv: 1205.2212] [INSPIRE].

[66] J. Polchinski and M.B. Wise, The Electric Dipole Moment of the Neutron in Low-Energy Supergravity, Phys. Lett. B 125 (1983) 393 [INSPIRE].

[67] A. De Rujula, M.B. Gavela, O. Pene and F.J. Vegas, Even larger contributions to the neutron electric dipole moment, Phys. Lett. B 245 (1990) 640 [INSPIRE]. 
[68] K. Fuyuto, J. Hisano, N. Nagata and K. Tsumura, QCD Corrections to Quark (Chromo)Electric Dipole Moments in High-scale Supersymmetry, JHEP 12 (2013) 010 [arXiv: 1308.6493] [INSPIRE].

[69] S. Dimopoulos and L.J. Hall, Baryogenesis at the MeV Era, Phys. Lett. B 196 (1987) 135 [INSPIRE].

[70] Super-Kamiokande collaboration, K. Abe et al., The Search for $n-\bar{n}$ oscillation in Super-Kamiokande I, arXiv:1109.4227 [INSPIRE].

[71] M.D. Litos, A search for dinucleon decay into kaons using the Super-Kamiokande water Cherenkov detector, Ph.D. thesis, Boston University (2010) [INSPIRE].

[72] J.L. Goity and M. Sher, Bounds on delta $B=1$ couplings in the supersymmetric standard model, Phys. Lett. B 346 (1995) 69 [Erratum ibid. B 385 (1996) 500] [hep-ph/9412208] [INSPIRE].

[73] T. Moroi, Effects of the gravitino on the inflationary universe, hep-ph/9503210 [INSPIRE].

[74] R.D. Peccei and H.R. Quinn, CP Conservation in the Presence of Instantons, Phys. Rev. Lett. 38 (1977) 1440 [INSPIRE].

[75] R.D. Peccei and H.R. Quinn, Constraints Imposed by CP Conservation in the Presence of Instantons, Phys. Rev. D 16 (1977) 1791 [InSPIRE].

[76] J.E. Kim, Light Pseudoscalars, Particle Physics and Cosmology, Phys. Rept. 150 (1987) 1 [INSPIRE].

[77] H.-Y. Cheng, The Strong CP Problem Revisited, Phys. Rept. 158 (1988) 1 [INSPIRE].

[78] J.E. Kim and G. Carosi, Axions and the Strong CP Problem, Rev. Mod. Phys. 82 (2010) 557 [arXiv:0807.3125] [INSPIRE].

[79] A. Ringwald, Exploring the Role of Axions and Other WISPs in the Dark Universe, Phys. Dark Univ. 1 (2012) 116 [arXiv:1210.5081] [INSPIRE].

[80] M. Kawasaki and K. Nakayama, Axions: Theory and Cosmological Role, Ann. Rev. Nucl. Part. Sci. 63 (2013) 69 [arXiv:1301.1123] [InSPIRE].

[81] K.S. Jeong and F. Takahashi, Light Higgsino from Axion Dark Radiation, JHEP 08 (2012) 017 [arXiv: 1201.4816] [inSPIRE].

[82] K.S. Jeong and F. Takahashi, Axionic Co-genesis of Baryon, Dark Matter and Dark Radiation, JHEP 04 (2013) 121 [arXiv: 1302.1486] [INSPIRE].

[83] M. Cicoli, J.P. Conlon and F. Quevedo, Dark Radiation in LARGE Volume Models, Phys. Rev. D 87 (2013) 043520 [arXiv: 1208.3562] [INSPIRE].

[84] T. Higaki and F. Takahashi, Dark Radiation and Dark Matter in Large Volume Compactifications, JHEP 11 (2012) 125 [arXiv:1208.3563] [INSPIRE].

[85] T. Higaki, K. Nakayama and F. Takahashi, Moduli-Induced Axion Problem, JHEP 07 (2013) 005 [arXiv: 1304.7987] [INSPIRE].

[86] E.J. Chun, D. Comelli and D.H. Lyth, The Abundance of relativistic axions in a flaton model of Peccei-Quinn symmetry, Phys. Rev. D 62 (2000) 095013 [hep-ph/0008133] [INSPIRE].

[87] K. Ichikawa, M. Kawasaki, K. Nakayama, M. Senami and F. Takahashi, Increasing effective number of neutrinos by decaying particles, JCAP 05 (2007) 008 [hep-ph/0703034] [INSPIRE]. 
[88] K. Choi and K.S. Jeong, String theoretic QCD axion with stabilized saxion and the pattern of supersymmetry breaking, JHEP 01 (2007) 103 [hep-th/0611279] [INSPIRE].

[89] J.P. Conlon, The QCD axion and moduli stabilisation, JHEP 05 (2006) 078 [hep-th/0602233] [INSPIRE].

[90] J.P. Conlon, F. Quevedo and K. Suruliz, Large-volume flux compactifications: Moduli spectrum and D3/D7 soft supersymmetry breaking, JHEP 08 (2005) 007 [hep-th/0505076] [INSPIRE].

[91] R. Blumenhagen, S. Moster and E. Plauschinn, Moduli Stabilisation versus Chirality for MSSM like Type IIB Orientifolds, JHEP 01 (2008) 058 [arXiv:0711.3389] [INSPIRE]. 